\title{
Magnetic order in orbital models of the iron pnictides
}

\author{
P. M. R. Brydon, ${ }^{1, \text { * }}$ Maria Daghofer, ${ }^{2,}$ † and Carsten Timm ${ }^{1,}$, \\ ${ }^{1}$ Institut für Theoretische Physik, Technische Universität Dresden, 01062 Dresden, Germany \\ ${ }^{2}$ IFW Dresden, P.O. Box 270116, 01171 Dresden, Germany
}

(Dated: July 9, 2021)

\begin{abstract}
We examine the appearance of the experimentally-observed stripe spin-density-wave magnetic order in five different orbital models of the iron pnictide parent compounds. A restricted meanfield ansatz is used to determine the magnetic phase diagram of each model. Using the random phase approximation, we then check this phase diagram by evaluating the static spin susceptibility in the paramagnetic state close to the mean-field phase boundaries. The momenta for which the susceptibility is peaked indicate in an unbiased way the actual ordering vector of the nearby meanfield state. The dominant orbitally resolved contributions to the spin susceptibility are also examined to determine the origin of the magnetic instability. We find that the observed stripe magnetic order is possible in four of the models, but it is extremely sensitive to the degree of the nesting between the electron and hole Fermi pockets. In the more realistic five-orbital models, this order competes with a strong-coupling incommensurate state which appears to be controlled by details of the electronic structure below the Fermi energy. We conclude by discussing the implications of our work for the origin of the magnetic order in the pnictides.
\end{abstract}

PACS numbers: 71.10.Fd, 74.70.Xa, 75.10.Lp

\section{INTRODUCTION}

The origins of high- $T_{c}$ superconductivity is one of the most important problems in contemporary condensed matter physics. There are now two main material classes in which such a state occurs: the cuprates, and the recently-discovered iron pnictides 12 The latter have therefore been the subject of an intensive research effort over the past few years, spurred by the hope to gain insight into the mechanisms of the unconventional superconductivity in both families.

The proximity of antiferromagnetic (AFM) and superconducting states in the phase diagram of the pnictides and the cuprates hints that exchange of virtual spin fluctuations is responsible for the pairing in both systems. ${ }^{3}$ Although this already provides strong motivation to study the magnetism of the pnictides, the unusual magnetic properties are of interest in their own right.7/8 It is now well established that the undoped cuprates are AFM Mott insulators ${ }^{6}$ In contrast, there is convincing evidence that the iron pnictide parent compounds $R \mathrm{FeAsO}$ and $A \mathrm{Fe}_{2} \mathrm{As}_{2}$ ( $R$ and $A$ are rare-earth and alkaline-earth elements, respectively) display a metallic spin-density-wave (SDW) state $\frac{78}{78}$ the relatively low value of the ordered moment ${ }^{5 \mid 9}$ and the appearance of incommensurate magnetism with doping, $\frac{10}{10}$ the presence of several electron and hole Fermi surface above $T_{N}$, and their significant reconstruction in the AFM state, as revealed by angle resolved photoemission spectroscopy (ARPES) and quantum oscillation experiments, 11 the compounds continue to show metallic transport properties below $T_{N}, \sqrt{14}[16$ and spectroscopic measurements suggest intermediate correlation strengths $17-19$

The microscopic description of the AFM state of the parent compounds in the cuprates and the pnictides will therefore be qualitatively different. While the $t-J$ model gives a good account of the former, $[6$ there is currently no generally-accepted model of the latter. By treating the Fe-As planes as a lattice of localized moments, several authors $21 \sqrt{24}$ have obtained good agreement with neutronscattering data.25 Although such a picture may be appropriate for the more strongly correlated iron chalcogenides, $\frac{8 \sqrt{20}}{{ }^{2}}$ it is difficult to reconcile with the experimental evidence for a moderately correlated metallic system in the 1111 and 122 pnictides. A more promising starting point for the pnictides is the prediction of $a b$ initio calculations that the nesting between the electron and hole Fermi pockets derived from the Fe $3 d$ orbitals is responsible for the SDW ${ }^{26 / 27}$ Theoretical work based on this scenario can be divided into two schools of thought: those which argue that only the nesting is important, 28 39 and those which also attempt to account for the complicated orbital structure of the Fermi surfaces $\$ 40$

The first approach can be dubbed the "excitonic" theory, as it is based upon the excitonic instability of a semimetal proposed in the $1960 \mathrm{~s}$.69 Within this scenario, the Coulomb attraction between electrons and holes about the nested electron and hole Fermi pockets, respectively, causes the condensation of interband electron-hole pairs (excitons). The condensed system is a density wave state with ordering vector $\mathbf{Q}$ equal to the nesting vector of the Fermi surfaces, with secondary interactions stabilizing a SDW ${ }^{29 / 70}$ Using suitably chosen phenomenological bands and effective interactions, the excitonic theory naturally explains the reconstruction of the Fermi surface and the continued metalicity below $T_{N}, \stackrel{33 \mid 3738}{1 t}$ it can reproduce the results of neutronscattering experiments,, 3436 and it appears to capture key aspects of the competition between superconductivity and the AFM state.29|31/33|35|39

Despite the success of the excitonic approach, it is unsatisfying as a microscopic model of the iron pnictides. 
Specifically, it ignores the complicated orbital structure of the different Fermi surfaces, $13 / 71$ 73 and the use of effective interactions obscures the roles of the various intra-ion interaction terms. 29133 Although the basic physics driving the AFM is expected to remain the same, it is desirable to include the orbital physics for a number of reasons: the variability in superconducting properties of the doped compounds suggests that small details of the electronic structure could play a significant role in the physics, 74 the likely presence of moderate correlations implies that some local physics should be included in theoretical models, ${ }^{75 \mid 76}$ while the real-space structure of the orbital wavefunctions, and the observed orbital reconstruction of the Fermi surface below $T_{N}, 13$ indicates a strong coupling of the orbital and magnetic degrees of freedom.

A large number of different orbital tightbinding models have therefore been proposed involving between two or five of the $\mathrm{Fe} 3 d$ orbitals, $42|43| 48|51| 52|54| 56|59| 60|64| 65 \mid 77+79$ or also including the As $4 p$ orbitals $45 \mid 80$ and even orbitals from outside

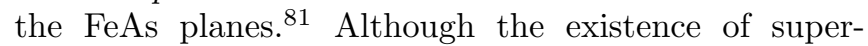
conductivity in these models has been extensively studied, 4044 46 $48|51| 53|56| 60|63| 64 \mid 66$ the magnetic behaviour remains rather poorly understood. The most popular approach to the appearance of AFM order in these models is to examine the ground state using a standard mean-field ansatz that allows for at most twosite magnetic unit cells, $\frac{47|50| 52|54| 58|59| 61 \mid 64}{6 e r y}$ recently, this has been generalized to a Gutzwiller mean-field theory! ${ }^{65 \mid 68}$ As all of the orbital models have a rather complicated electronic structure, however, it is by no means certain that such phases have the lowest free energy, i.e. states with larger unit cells or incommensurate ordering vectors cannot be a priori excluded. Indeed, in several cases more advanced techniques have been used to study the magnetic properties with mixed results: whereas in some models the findings are consistent with the usual mean-field ansatz, 49/57/60|64/67 striking


and robustness of the experimentally-observed stripe AFM order in these models at parent compound filling therefore remains an important open problem, as it is questionable to use them to examine the superconductivity of the doped system if they do not give the correct magnetic behaviour of the parent compound.

It is hence desirable to examine the magnetic properties of these orbital models in an unbiased way. This can be achieved by examining the behaviour of the static spin susceptibility $\chi_{s}(\mathbf{q}, \omega=0)$ in the paramagnetic $(\mathrm{PM})$ state: as the temperature is lowered towards the critical temperature $T_{\text {mag }}$ of a magnetically-ordered phase, the susceptibility will diverge at the ordering vector $\mathbf{q}=\mathbf{Q}$. Practically, we can implement this scheme in a weakcoupling approach by first calculating the phase diagram of the model using a two-site mean-field ansatz, and then examining the location of the highest peaks in the random-phase approximation (RPA) spin susceptibility at a temperature just above $T_{\text {mag }}$ to check the validity of the low-temperature mean-field state. Clues to the origin of the magnetic instability can be extracted from the dominant contributions to the total static susceptibility from the orbitally resolved susceptibilities.

It is impossible to study each of the very many different orbital models proposed for the pnictides, and so in this paper we will restrict ourselves to five models: the two-orbital model of Raghu et al., 43 the three-orbital model of Daghofer et al., 59 the four-orbital model of $\mathrm{Yu}$ et al., 54 and the five-orbital models of Kuroki et al.,, 40 and Graser et al. ${ }^{48}$ The main goal of this paper is to verify the appearance and explore the robustness of the stripe AFM order in the weak-coupling limit in these very different models, which have been selected as a representative sample of the different levels of sophistication available in the literature. By this survey we not only hope to test the suitability of these models and the weak-coupling theory to describe the pnictide parent compounds, but also to gain insight into the origin of the AFM state and the features of the electronic structure that enhance or suppress it. We additionally hope to identify an orbital model which gives the required AFM state and is also consistent with key results of $a b$ initio calculations and ARPES data. Finally, the role of Hartree shifts in renormalizing the band structure and the implications for the magnetism is examined.

Particular attention in our analysis will be paid to the five-orbital models of Kuroki et al. and Graser et al. because they are currently the most intensively-studied, the most sophisticated, and likely most accurate as they both result from fits to $a b$ initio band structures of LaFeAsO. We note that other five-orbital models of LaFeAsO were proposed in Ref. 78 and Ref. 60, but we will not examine them here. The latter is very similar to Kuroki et al.'s model, and therefore we neglect it here in the interests of brevity. The former is quite different to Kuroki et al.'s and Graser et al.'s models as it was not the result of fitting to $a b$ initio calculations and does not include higher-order hopping terms. This leads to very strong ellipticity of the electron Fermi pockets, which substantially reduces Fermi-surface nesting and should significantly alter the magnetic fluctuations. Since the focus of our paper is on nesting and the resulting magnetic instabilities, we do not consider this model.

The structure of the paper is as follows. In section II we outline the theoretical basis of our analysis by discussing the general form of the Hamiltonian, the meanfield decoupling scheme, and the construction of the spin susceptibility within RPA. We then proceed to a systematic analysis of the magnetic order in the five models in section III. In section IV we compare and contrast the ordering properties of the models, and consider the implications for the pnictides. We conclude with a summary in section $\mathrm{V}$. 


\section{THEORY}

The general Hamiltonian for the orbital models is written $H=H_{0}+H_{I}$. The non-interacting Hamiltonian $H_{0}$ is given by

$$
H_{0}=\sum_{\mathbf{k}} \sum_{\sigma} \sum_{\nu, \mu} T_{\nu, \mu}(\mathbf{k}) d_{\mathbf{k}, \nu, \sigma}^{\dagger} d_{\mathbf{k}, \mu, \sigma}
$$

where $d_{\mathbf{k}, \nu, \sigma}^{\dagger}\left(d_{\mathbf{k}, \nu, \sigma}\right)$ is the creation (annihilation) operator for a spin $\sigma$ electron of momentum $\mathbf{k}$ in the orbital $\nu . T_{\nu, \mu}(\mathbf{k})$ are the kinetic energy matrix elements for an effective tight-binding model defined in the single-Fe unit cell of the two-dimensional Fe-As planes.

In all the proposed models, only on-site terms are included in the interaction Hamiltonian

$$
\begin{aligned}
H_{I}= & U \sum_{\mathbf{i}} \sum_{\nu} n_{\mathbf{i}, \nu, \uparrow} n_{\mathbf{i}, \nu, \downarrow}+V \sum_{\mathbf{i}} \sum_{\nu \neq \mu} \sum_{\sigma, \sigma^{\prime}} n_{\mathbf{i}, \nu, \sigma} n_{\mathbf{i}, \mu, \sigma^{\prime}} \\
& -J \sum_{\mathbf{i}} \sum_{\nu \neq \mu} \mathbf{S}_{\mathbf{i}, \nu} \cdot \mathbf{S}_{\mathbf{i}, \mu} \\
& +J^{\prime} \sum_{\mathbf{i}} \sum_{\nu \neq \mu} d_{\mathbf{i}, \nu, \uparrow}^{\dagger} d_{\mathbf{i}, \nu, \downarrow}^{\dagger} d_{\mathbf{i}, \mu, \downarrow} d_{\mathbf{i}, \mu, \uparrow}
\end{aligned}
$$

The number and spin operator for the orbital $\nu$ at site $\mathbf{i}$ are defined as $n_{\mathbf{i}, \nu, \sigma}=d_{\mathbf{i}, \nu, \sigma}^{\dagger} d_{\mathbf{i}, \nu, \sigma}$ and $\mathbf{S}_{\mathbf{i}, \nu}=$ $\frac{1}{2} \sum_{\varsigma, \varsigma^{\prime}} d_{\mathbf{i}, \nu, \varsigma}^{\dagger} \boldsymbol{\sigma}_{\varsigma, \varsigma^{\prime}} d_{\mathbf{i}, \nu, \varsigma^{\prime}}$, respectively, where $\boldsymbol{\sigma}_{\varsigma, \varsigma^{\prime}}$ is the vector of the Pauli spin matrices. The matrix elements of the kinetic energy of all the models considered here were explicitly constructed to obey the orbital and lattice symmetries of the Fe-As planes, which has been shown to be possible for a one-Fe unit cell in 2-dimensional models. ${ }^{[72}$ Even though the interaction terms are purely onsite, some restrictions on the parameters still arise from the requirement that it should preserve the symmetries of the orbital space. The orientation of the axes of the coordinate system used to define the orbitals must not change the symmetries of the Hamiltonian, which implies $J=J^{\prime}$ and $V=(2 U-5 J) / 4.82$

For the sake of brevity we only present results for Hund's rule coupling $J / U=0.25$. Although this ratio is at the upper limit of those employed in the literature, it is not unreasonable. Screening can considerably reduce the effective Coulomb interactions $U$ and $V$ from their atomic values, which suggests rather large ratios of $J / U$. On the other hand, selecting $J \leq U / 3$ ensures that an electron added to an undoped site pays more energy to Coulomb repulsion than it can win from Hund's rule, i.e. that the onsite interaction energy suppresses charge fluctuations rather than enhancing them. Furthermore, a mean-field study of a number of models has recently found that the ordered moment and the reconstructed Fermi surface in the $T=0 \mathrm{~K}$ AFM state is in good agreement with experiment for our choice of $J / U \underline{64}$

\section{A. Mean-field analysis}

When constructing the mean-field phase diagrams of the orbital models, we adopt the frequently-employed ansatz $\frac{54|58| 59|61| 64 \mid 83}{2}$

$$
\left\langle n_{\mathbf{i}, \nu, \sigma}\right\rangle=n_{\nu}+\frac{\sigma}{2} e^{i \mathbf{Q} \cdot \mathbf{r}_{\mathbf{i}}} m_{\nu}
$$

Note that this neglects the possibility of inter-orbital averages $\left\langle d_{\mathbf{i}, \nu, \sigma}^{\dagger} d_{\mathbf{i}, \mu, \sigma}\right\rangle(\nu \neq \mu)$, which have been included in some studies ${ }^{4752}$ We restrict ourselves to ferromagnetic (FM) and AFM phases with a two-site unit cell, corresponding to the ordering vectors $\mathbf{Q}=(0,0), \mathbf{Q}=(\pi, 0)$ and $\mathbf{Q}=(\pi, \pi)$ in $(3)$, respectively. The $\mathbf{Q}=(\pi, 0)$ AFM state gives the magnetic order found in the pnictide parent compounds. Upon decoupling the interaction term $H_{I}$, we obtain the mean-field Hamiltonian

$$
\begin{aligned}
H_{\mathrm{MF}}= & H_{0}+\sum_{\mathbf{k}} \sum_{\nu} \sum_{\sigma} \epsilon_{\nu} d_{\mathbf{k}, \nu, \sigma}^{\dagger} d_{\mathbf{k}, \nu, \sigma} \\
& -\frac{1}{2} \sum_{\mathbf{k}} \sum_{\nu} \sum_{\sigma} \sigma \eta_{\nu} d_{\mathbf{k}+\mathbf{Q}, \nu, \sigma}^{\dagger} d_{\mathbf{k}, \nu, \sigma}+N C,
\end{aligned}
$$

where $N$ is the number of lattice sites and

$$
\begin{aligned}
\epsilon_{\nu}= & U n_{\nu}+(2 U-5 J) \sum_{\mu \neq \nu} n_{\mu} \\
\eta_{\nu}= & U m_{\nu}+J \sum_{\mu \neq \nu} m_{\mu} \\
C= & -U \sum_{\nu} n_{\nu}^{2}+\frac{U}{4} \sum_{\nu} m_{\nu}^{2}-(2 U-5 J) \sum_{\mu \neq \nu} n_{\nu} n_{\mu} \\
& +\frac{J}{4} \sum_{\mu \neq \nu} m_{\nu} m_{\mu}
\end{aligned}
$$

Although the Hartree shifts $\epsilon_{\nu}$ can have rather large magnitude, only the relative shifts $\widetilde{\epsilon}_{\nu}=(5 J-U)\left(n_{\nu}-\right.$ $\left.\min \left\{n_{\mu}\right\}\right)$ are important when working at fixed doping. These relative shifts are much smaller than the bare shifts $\epsilon_{\nu}$. Note that not all mean-field studies include the Hartree shifts. ${ }^{47 / 52}$ The influence of Hartree shifts on the phase diagram will be discussed in section IVB.

For given temperature $T$ and interaction constants $U$ and $J$, the stable mean-field state is determined by the standard iterative diagonalization technique, where the condition of constant charge density

$$
\sum_{\nu} n_{\nu}= \begin{cases}1 & \text { for } 2 \text { orbitals } \\ 2 & \text { for } 3 \text { and } 4 \text { orbitals } \\ 3 & \text { for } 5 \text { orbitals }\end{cases}
$$

is enforced at each iteration step. Upon convergence, the free energy per site is calculated. Comparing the free energy for each mean-field ansatz allows us to determine the stable state of the system. 


\section{B. The magnetic susceptibility}

We define the spin susceptibility by

$$
\begin{aligned}
& \chi^{j j^{\prime}}\left(\mathbf{q}, i \omega_{n}\right) \\
& =\frac{1}{N} \sum_{\nu, \mu} \int_{0}^{\beta} d \tau e^{i \omega_{n} \tau}\left\langle T_{\tau} S_{\nu}^{j}(\mathbf{q}, \tau) S_{\mu}^{j^{\prime}}(-\mathbf{q}, 0)\right\rangle,
\end{aligned}
$$

where the Fourier-transformed spin operator $S_{\nu}^{j}(\mathbf{q})$ is related to the spin operator at site $\mathbf{i}$ by

$$
S_{\mathbf{i}, \nu}^{j}=\frac{1}{\sqrt{N}} \sum_{\mathbf{q}} S_{\nu}^{j}(\mathbf{q}) e^{-i \mathbf{q} \cdot \mathbf{r}_{\mathbf{i}}} .
$$

The total spin susceptibility is hence written as

$$
\chi_{s}\left(\mathbf{q}, i \omega_{n}\right)=\sum_{j=x, y, z} \chi^{j j}\left(\mathbf{q}, i \omega_{n}\right) .
$$

To obtain the static spin susceptibilities we make the analytic continuation $i \omega_{n} \rightarrow \omega+i 0^{+}$and then take the limit $\omega=0$. Note that it is not necessary to regularize the static susceptibility by assuming a non-zero imaginary part of the frequency. Because of the spin-rotation invariance of the PM state, we can express $\chi_{s}(\mathbf{q}, \omega=0)$ in terms only of the static transverse susceptibility

$$
\chi_{s}(\mathbf{q}, \omega=0)=\frac{3}{2} \chi^{-+}(\mathbf{q}, \omega=0) \text {. }
$$

Henceforth we will only be concerned with $\chi^{-+}(\mathbf{q}, \omega=0)$ as this contains all relevant information on the magnetic ordering vector.

We calculate the transverse spin susceptibility using the RPA. Introducing the generalized transverse susceptibilities

$$
\begin{aligned}
& \chi_{\nu, \nu^{\prime}, \mu, \mu^{\prime}}^{-+}\left(\mathbf{q}, i \omega_{n}\right) \\
& =\frac{1}{N} \sum_{\mathbf{k}, \mathbf{k}^{\prime}} \int_{0}^{\beta} d \tau e^{i \omega_{n} \tau} \\
& \quad \times\left\langle T_{\tau} d_{\mathbf{k}+\mathbf{q}, \nu, \downarrow}^{\dagger}(\tau) d_{\mathbf{k}, \nu^{\prime}, \uparrow}(\tau) d_{\mathbf{k}^{\prime}-\mathbf{q}, \mu, \uparrow}^{\dagger}(0) d_{\mathbf{k}^{\prime}, \mu^{\prime}, \downarrow}(0)\right\rangle,
\end{aligned}
$$

we write the total transverse susceptibility

$$
\chi^{-+}\left(\mathbf{q}, i \omega_{n}\right)=\sum_{\nu, \mu} \chi_{\nu, \nu, \mu, \mu}^{-+}\left(\mathbf{q}, i \omega_{n}\right) .
$$

Summation of the ladder diagrams yields a Dyson equation for the generalized susceptibilities

$$
\begin{aligned}
& \chi_{\nu, \nu^{\prime}, \mu, \mu^{\prime}}^{-+}\left(\mathbf{q}, i \omega_{n}\right) \\
& =\chi_{\nu, \nu^{\prime}, \mu, \mu^{\prime}}^{-+\left(\mathbf{q}, i \omega_{n}\right)} \\
& \quad+\chi_{\nu, \nu^{\prime}, a, b}^{-+(0)}\left(\mathbf{q}, i \omega_{n}\right) V_{a, b, c, d} \chi_{c, d, \mu, \mu^{\prime}}^{-+}\left(\mathbf{q}, i \omega_{n}\right),
\end{aligned}
$$

where the non-zero elements of $V_{a, b, c, d}$ are

$$
V_{a, a, a, a}=U
$$

$$
\begin{aligned}
& V_{a, a, b, b}=J \\
& V_{a, b, b, a}=U-2 J \\
& V_{a, b, a, b}=J
\end{aligned}
$$

and we assume $a \neq b$. The mean-field susceptibilities $\chi_{\nu, \nu^{\prime}, \mu, \mu^{\prime}}^{-+(0)}\left(\mathbf{q}, i \omega_{n}\right)$ can be explicitly written as

$$
\begin{aligned}
& \chi_{\nu, \nu^{\prime}, \mu, \mu^{\prime}}^{-+\left(\mathbf{q}, i \omega_{n}\right)} \\
& =-\frac{1}{N} \sum_{\mathbf{k}} \sum_{s, s^{\prime}} u_{s, \nu^{\prime}}(\mathbf{k}) u_{s, \mu}^{*}(\mathbf{k}) u_{s^{\prime}, \mu^{\prime}}(\mathbf{k}+\mathbf{q}) u_{s^{\prime}, \nu}^{*}(\mathbf{k}+\mathbf{q}) \\
& \times \frac{n_{F}\left(E_{s, \mathbf{k}}\right)-n_{F}\left(E_{s^{\prime}, \mathbf{k}+\mathbf{q}}\right)}{E_{s, \mathbf{k}}-E_{s^{\prime}, \mathbf{k}+\mathbf{q}}-i \omega_{n}},
\end{aligned}
$$

where $n_{F}(E)$ is the Fermi function, $E_{s, \mathbf{k}}$ are the eigenvalues of $H_{\mathrm{MF}}$ (4), and the coefficients $u_{s, \nu}(\mathbf{k})$ transform the diagonalizing annihilation operators $\gamma_{s, \mathbf{k}}$ corresponding to the eigenvalues $E_{s, \mathbf{k}}$ into the orbital basis, i.e. $d_{\nu, \mathbf{k}}=\sum_{s} u_{s, \nu}(\mathbf{k}) \gamma_{s, \mathbf{k}}$.

Apart from the total transverse spin susceptibility, it is also interesting to consider the dominant contributions to the sum (13). It is convenient to define $\chi_{\nu, \mu}^{-+}\left(\mathbf{q}, i \omega_{n}\right) \equiv \chi_{\nu, \nu, \mu, \mu}^{-+}\left(\mathbf{q}, i \omega_{n}\right)$. These orbitally resolved susceptibilities can provide important insight into the regions of the Fermi surface most strongly involved in the magnetic order. We adopt the following enumeration of the orbitals: $\nu=1(x z), \nu=2(y z), \nu=3(x y), \nu=4$ $\left(x^{2}-y^{2}\right)$, and $\nu=5\left(3 z^{2}-r^{2}\right)$.

\section{RESULTS}

In this section we present a systematic analysis of the magnetic behaviour of the two-orbital model of Raghu et $a l$.43] (section III A), the three-orbital model of Daghofer et al ${ }^{59}$ (section III B), the four-orbital model of $\mathrm{Yu}$ et al ${ }^{54}$ (section III C), and the five-orbital models of Kuroki


In all cases, we first determine the mean-field phase diagram using the method outlined in section II A and then calculate the susceptibilities at several points in the PM state lying close to the boundary of the ordered phases. We emphasize that we do not seek to construct the true mean-field phase diagram of the orbital models, but rather to determine the appropriateness of the mean-field ansatz 3 .

All calculations (i.e. the phase diagram and the meanfield susceptibilities) were performed using a $400 \times 400 \mathrm{k}$ point mesh. In order to better distinguish the total and the orbitally resolved susceptibilities from one another, we use a different color scheme in the density plots of these quantities. We also note that the total static susceptibility is symmetric about the line $q_{x}=q_{y}$; if peaks in $\chi^{-+}(\mathbf{q}, \omega=0)$ are found off this line, for simplicity we will only mention the peak with $q_{x}>q_{y}$ in the discussion. 



(a)

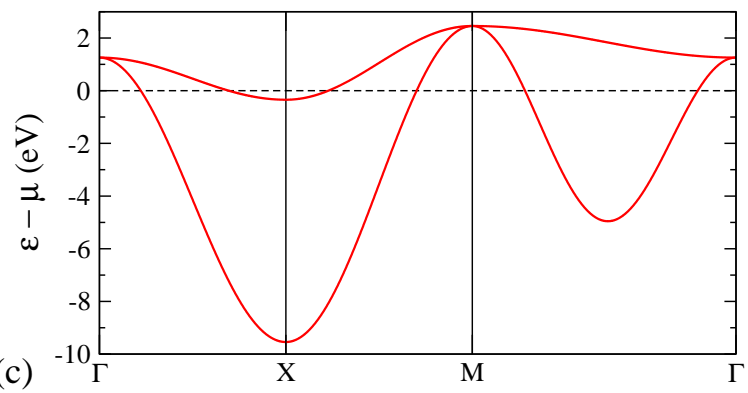

FIG. 1: (a) The Fermi surface of the two-orbital model of Raghu et al ${ }^{43}$ showing the dominant orbital contribution. (b) The orbital weight around the $\alpha$ and $\beta$ Fermi surfaces as a function of the winding angle $\theta$ measured with respect to the $y$-axis, taken in the anti-clockwise direction. The orbital weight around the $\gamma$ Fermi surface is not shown as it has almost complete $y z$ character. (c) The band structure along high-symmetry directions.

\section{A. The two-orbital model of Raghu et al.}

The two-orbital model of Raghu et al ${ }^{43}$ was one of the first attempts to model the pnictides from an orbital point of view, and also the first of a number of similar two-orbital models. $\frac{42152 / 55 / 77}{4}$ As it only keeps the $x z$ and $y z$ orbitals, it may be regarded as a minimal orbital model of the system. Although it is likely too simple to give quantitative agreement with experiment, it is nevertheless of interest to us as it qualitatively captures the variation of the orbital character about the Fermi surface and hence provides important clues to the ordering mechanism.

The Fermi surface and the band structure of the twoorbital model is shown in figure $1{ }^{84}$ As we shall see, the Fermi surface is somewhat unlike those of the more sophisticated models, specifically one of the $x z / y z$-derived hole pockets is located at $(\pi, \pi)$ instead of at $(0,0)$. This implies rather different magnetic properties compared to these other models, as the nesting of the hole pocket at $(\pi, \pi)$ with the electron pocket at $(0, \pi)$ allows both electron pockets to participate on an almost equal footing in the $(\pi, 0)$ AFM state.

The mean-field phase diagram of the two-orbital model is presented in figure 2. The $(\pi, 0)$ AFM state is stable

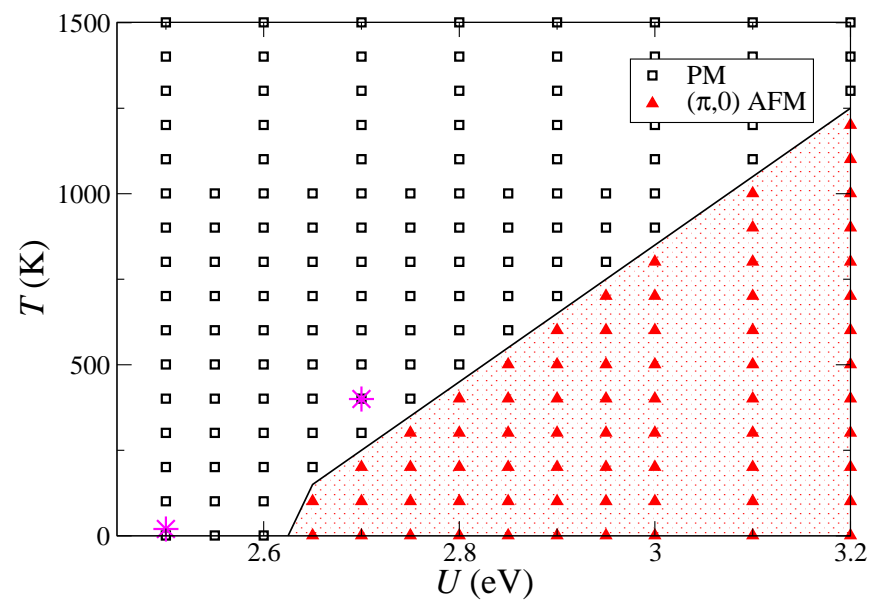

FIG. 2: The $T-U$ mean-field phase diagram of the two-orbital model of Raghu et al. ${ }^{43}$ The location of the symbols indicate a co-ordinate at which the free energy was evaluated, and the corresponding minimizing state. The shaded region approximately indicates the extent of the $(\pi, 0)$ AFM phase. We show the static susceptibility at the points indicated by the star symbols in figure 3

above a critical interaction strength $U_{c} \approx 2.65 \mathrm{eV}$, with the critical temperature increasing linearly with $U$ above this value. Our results are consistent with those of Ref. 50 for $J=0.1 U$. In a similar two-orbital model ${ }^{411}$ the meanfield ground state was found to be a superposition of $\mathbf{Q}_{x}=(\pi, 0)$ and $\mathbf{Q}_{y}=(0, \pi)$ stripe magnetic orders with mutually-perpendicular spin polarizations. Although our aim is to check the validity of the mean-field phase diagram by examining the spin susceptibility just above the mean-field ordering temperature, we are not able to determine whether such a two- $\mathbf{Q}$ state or the expected $(\pi, 0)$ AFM phase is realized: in both cases we will find a peak in the susceptibility at $(\pi, 0)$.

In figure 3 we show the static susceptibility at the points indicated in figure 2. At both points the susceptibility is sharply peaked at or very close to $(\pi, 0)$, reflecting the good nesting of the electron and hole pockets, and confirming the appropriateness of our mean-field ansatz. The absence of any other significant structure in the susceptibility implies that only scattering between the electron and hole pockets is important to the magnetic ordering. Examining the susceptibility at $\approx 150 \mathrm{~K}$ above the AFM transition temperature at a number of interaction strengths between $U=2.7 \mathrm{eV}$ and $3 \mathrm{eV}$, we find only minor quantitative differences in $\chi^{-+}(\mathbf{q}, \omega=0)$ within this range. At $U<U_{c}$ the magnetic response indicates weak incommensuration, with the susceptibility peaking at $\mathbf{q} \approx(0.95 \pi, 0)$ [see figure $3(\mathrm{a})$ ]. An incommensurate AFM phase is in fact realized at $U=2.6 \mathrm{eV}$ and $T=100 \mathrm{~K}$, as revealed by the observation of negative values of $\chi^{-+}(\mathbf{q}, \omega=0)$ here. The unrestricted real-space Hartree-Fock of Ref. 41 may have missed the weaklyincommensurate phase because the considered cluster 

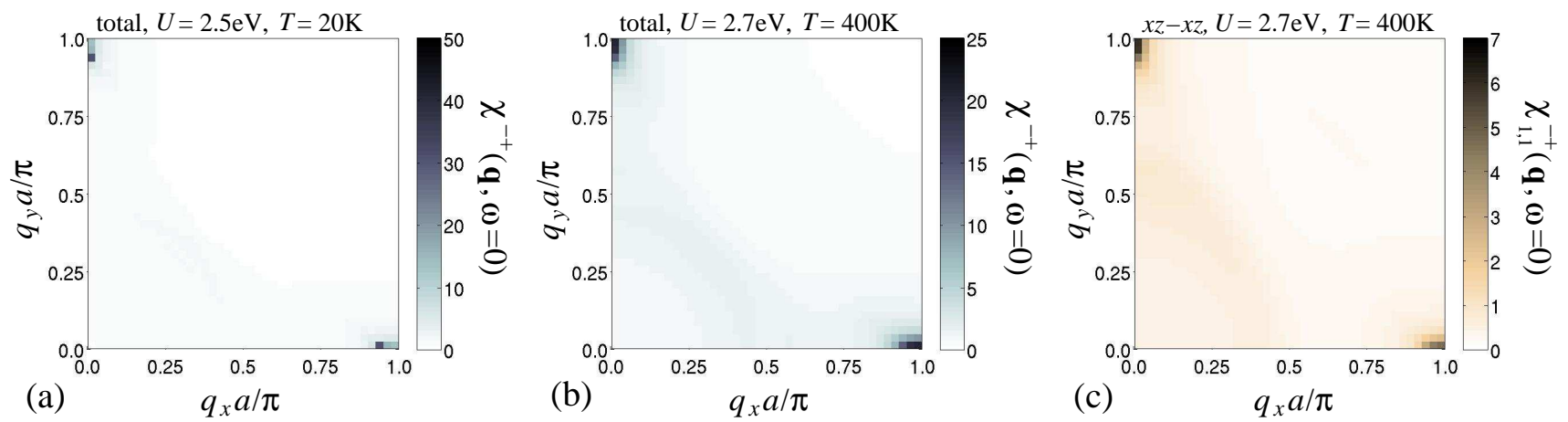

FIG. 3: The static transverse spin susceptibility of the two-orbital model of Raghu et al ${ }^{43}$ at (a) $U=2.5 \mathrm{eV}$ and $T=20 \mathrm{~K}$ and (b) $U=2.7 \mathrm{eV}$ and $T=400 \mathrm{~K}$. (c) The dominant contribution to the static susceptibility from the $x z-x z$ term at $U=2.7 \mathrm{eV}$ and $T=400 \mathrm{~K}$. Note the different scales used in each panel.

size was too small.

The dominant contribution to the static transverse susceptibility is from the $x z-x z$ and $y z-y z$ terms. $\chi_{1,1}^{-+}(\mathbf{q}, \omega=0)$ is shown in figure $3(\mathbf{c}) ; \chi_{2,2}^{-+}(\mathbf{q}, \omega=0)$ can be obtained from this by interchanging $q_{x}$ and $q_{y}$. We see that although there are definite peaks at both $(\pi, 0)$ and $(0, \pi)$, the latter peak is somewhat higher than the former. This implies that the $y z[x z]$ orbital is slightly more important to $(\pi, 0)[(0, \pi)]$ AFM order, consistent with the mean-field calculation in Ref. 50

\section{B. The three-orbital model of Daghofer et al.}

The three-orbital model of Daghofer et al ${ }^{[59}$ is the newest model studied in this work. In addition to the $x z$ and $y z$ orbitals, this model also includes the $x y$ orbital. Assuming that the magnetic order is driven by nesting of electron and hole Fermi surfaces, it is reasonable to neglect the $x^{2}-y^{2}$ and $3 z^{2}-r^{2}$ orbitals as ab initio calculations predict that they have very little weight at the Fermi energy.

The electronic structure of the three-orbital model is summarized in figure 4. The model reproduces key features of the Fermi surface predicted by ab initio calculations: there are two $x z / y z$-derived nearly circular hole pockets at the centre of the Brillouin zone, and a $y z / x y$ derived $[x z / y z$-derived] elliptical electron pocket centred at $\mathbf{k}=(\pi, 0)[\mathbf{k}=(0, \pi)]$. We note that the hole pockets have much greater effective mass than the electron pockets, in striking contrast to the two-orbital model discussed above.

The ground state phase diagram of the model as a function of $J$ and $U$ was mapped in Ref. 59 using a mean-field ansatz that also allowed for stripe or staggered orbital order in addition to FM and AFM states. At moderate- to strong-coupling, orbital ordered states were obtained; in the weak-coupling regime and $J=0.25 U$, however, the $(\pi, 0)$ AFM state without orbital order was found to be stable. The authors of Ref. 64 mapped the same phase
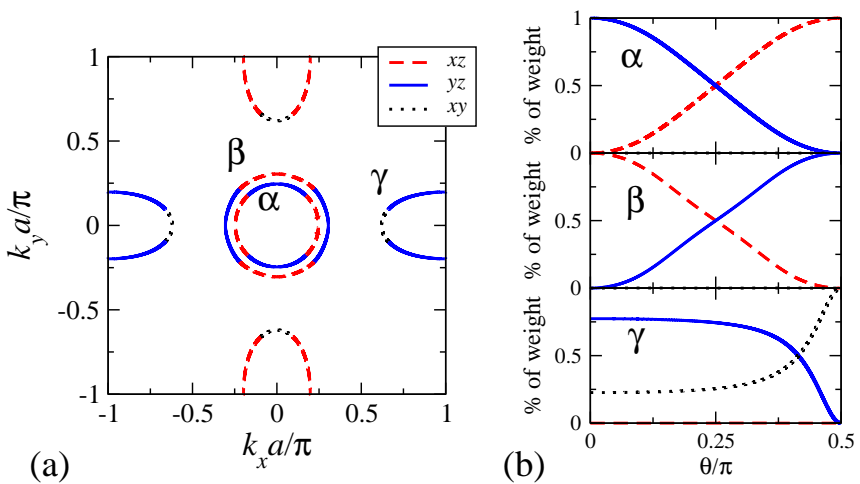

(c)



FIG. 4: (a) The Fermi surface of the three-orbital model of Daghofer et al. ${ }^{[59}$ showing the dominant orbital contribution. (b) The orbital weight around the labeled Fermi surfaces. (c) The band structure along high-symmetry directions.

diagram using a more restricted mean-field ansatz, but their RPA calculations suggest that the $(\pi, 0)$ AFM state is unstable towards incommensurate order. In figure 5 , we show the phase diagram in the $T-U$ plane.

Our results for the spin susceptibility are in agreement with Ref. 64, and we therefore conclude that the phase diagram figure 5 does not reflect the actual mean-field behaviour of the model. At relatively weak coupling the dominant magnetic response occurs in a ring of almost uniform height centred at $\mathbf{q}=(0,0)$ of radius $\approx 0.5 \pi$ and $0.4 \pi$, see figure 6(a) and (b) respectively. In the former 


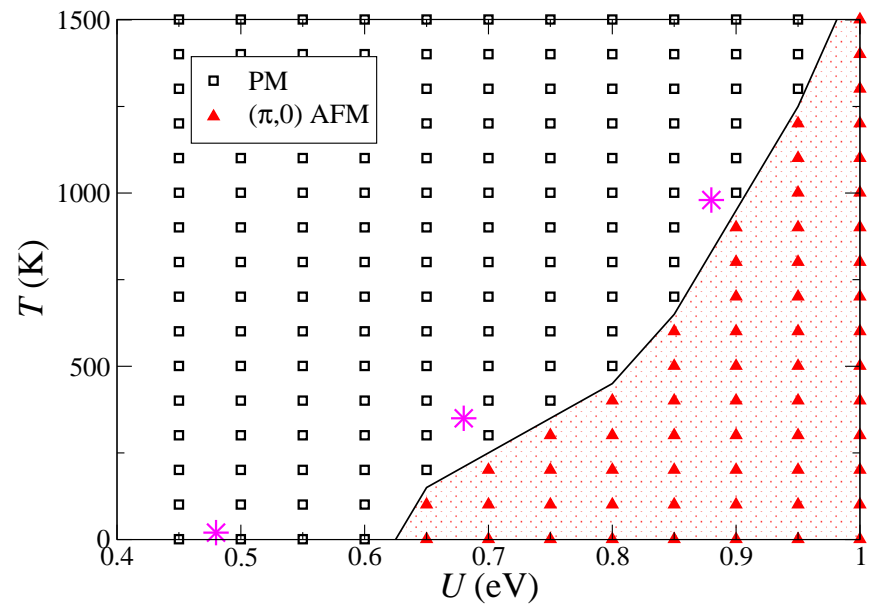

FIG. 5: The T-U mean-field phase diagram of the threeorbital model of Daghofer et al..59. The shaded region approximately indicates the extent of the $(\pi, 0)$ AFM phase. We show the static susceptibility at the points indicated by the star symbols in figure 6 .

case, we can make out another, fainter ring of radius $\approx 0.65 \pi$; the radii of these rings correspond exactly to twice the average radii of the hole pockets, indicating that the magnetic response is dominated by scattering from one side of the hole Fermi surfaces to the other. The smaller radius of the ring in the $U=0.68 \mathrm{eV}$ data reflects the reduction of the size of the heavy hole pockets by the increase of the chemical potential with temperature. In both of these figures, there is only a very small peak close to $(\pi, 0)$; this peak is barely visible in the $U=$ $0.68 \mathrm{eV}, T=350 \mathrm{~K}$ data. The form of the susceptibility changes significantly at higher $U$ : as shown in figure 6(c), two rather broad peaks develop at $\mathbf{q} \approx(0.75 \pi, 0)$. The origin of this behaviour is unclear, as this wavevector does not seem to correlate with any Fermi surface or band structure feature.

It is interesting to consider the consequence of shrinking the hole Fermi surfaces in this model, for example by electron-doping. We expect that this should also shrink the ring feature in the susceptibility, leading to significant weight close to $\mathbf{q}=0$, favouring $\mathrm{FM}$ order and triplet superconductivity. Such a situation might be relevant to the stochiometric superconductor LiFeAs, where ARPES reveals small heavy hole pockets which are poorly nested with electron Fermi surfaces ${ }^{85}$ A model for this compound which captures these salient band structure features was recently shown to display FM order and triplet superconductivity in the weak-coupling limit. ${ }^{79}$ Intriguingly, unpublished NMR results on single crystals of LiFeAs report that there is no suppression of the Knight shift below $T_{c}$ for magnetic fields perpendicular to the crystal $c$ axis, strongly suggesting triplet pairing. ${ }^{86}$

We note that there are several alternative three-orbital models which display an $x y$-dominated hole pocket at the M point. ${ }^{4453}$ A similar analysis to that performed here has been carried out for such a three-orbital model 62 and the susceptibility was found to be peaked at an incommensurate wavevector close to $(\pi, 0)$. More recently, Zhou and Wang studied a three-orbital model of LaFeAsO using Gutzwiller mean-field theory restricted to two-site magnetic unit cells and found significant deviations from the usual Hartree-Fock approach. $[65$

\section{The four-orbital model of Yu et al.}

The four-orbital model of $\mathrm{Yu}$ et al .54 includes all but the $3 z^{2}-r^{2}$ orbital. Although neither the $x^{2}-y^{2}$ or the $3 z^{2}-r^{2}$ orbitals significantly contribute to the Fermi surface, the former is filled at lower doping levels. This suggests that the $3 z^{2}-r^{2}$ orbital will be less relevant to the magnetism, $\frac{51}{2}$, justifying its exclusion from this model.

As shown in figure 7, the structure of the Fermi surface is broadly similar to that of the three-orbital model, with elliptical electron Fermi pockets at the X points, and two nearly circular hole pockets with much greater effective mass at the $\Gamma$ point. Note, however, that the electron pockets are rotated and have reversed dominant orbital content (i.e. $x z \leftrightarrow y z$ ), while each Fermi surface is smaller. These are likely relatively superficial differences: the orientation of the electron pockets is correct in the (physical) two-Fe Brillouin zone, while the size of the Fermi pockets can be tuned through the tight-binding parameters. More seriously, however, the model contradicts some key predictions of ab initio calculations: the $x y$ weight at the Fermi surface is heavily suppressed, 71772 and only a single band contributes to the electron Fermi pockets. 26171

Our mean-field phase diagram agrees with the $T=0 \mathrm{~K}$ analysis of $\mathrm{Yu}$ et al.: we find a low-temperature $(\pi, 0)$ AFM state for $1.9 \mathrm{eV} \lesssim U \lesssim 2.65 \mathrm{eV}$, while above a critical $U_{c} \approx 2.65 \mathrm{eV}$ the ground state is the $(\pi, \pi) \mathrm{AFM}$ which has a very high ordering temperature $(>3000 \mathrm{~K})$. It is possible that the strong-coupling $(\pi, 0)$ AFM state found in Ref. 54 has lower free energy for $U>4 \mathrm{eV}$, but at these coupling strengths the AFM states are insulating and hence irrelevant to the magnetic ordering in the pnictides.

In figure 9 we show the static transverse susceptibility at the points indicated in the phase diagram figure 8 . The low- $U$ results [figure 9 (a) and (b)] are strongly reminiscent of the two-orbital model. For $U=1.8 \mathrm{eV}$ and $T=20 \mathrm{~K}$, just below the critical coupling strength of the $(\pi, 0)$ AFM state, $\chi^{-+}(\mathbf{q}, \omega=0)$ is peaked at $\mathbf{q} \approx(0.95 \pi, 0)$. Closer to the $(\pi, 0)$ AFM phase there is evidence that an ordered incommensurate state is realized. At $U=2.1 \mathrm{eV}$ and $T=400 \mathrm{~K}$, we find a sharp peak exactly at $\mathbf{q}=(\pi, 0)$. These peaks are clearly derived from the very good nesting of the electron and hole Fermi surfaces. This conclusion is supported by examination of the dominant contributions to the peak at $(\pi, 0)$ in figure $9(\mathrm{~d}-\mathrm{f})$ : the $x z-x z$ term has the largest value, 

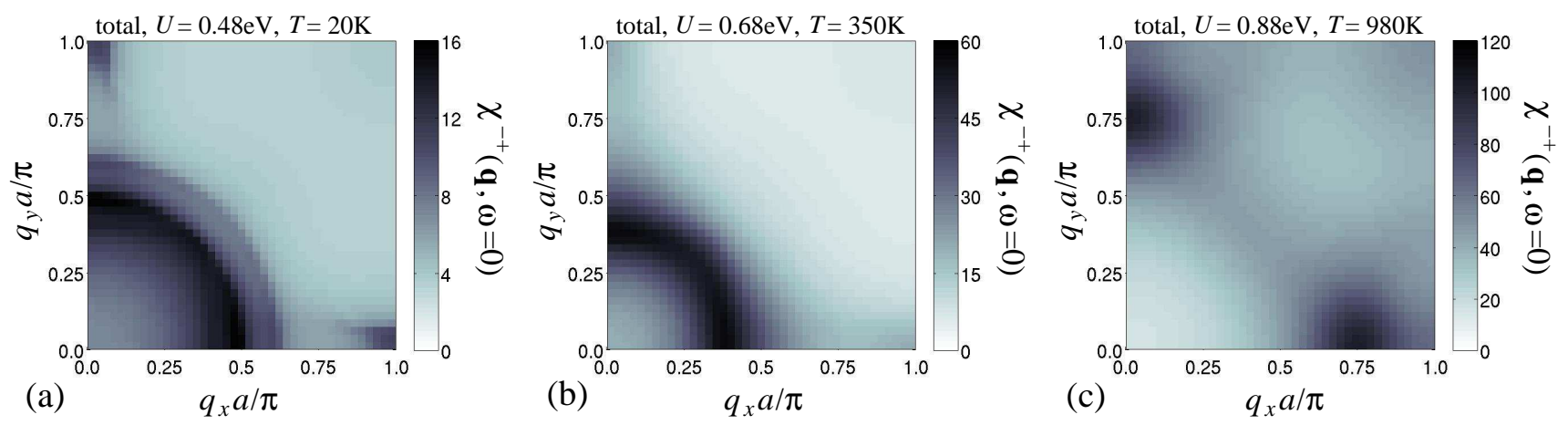

FIG. 6: The static transverse spin susceptibility of the three-orbital model of Daghofer et al. ${ }^{59}$ at $(\mathrm{a}) U=0.48 \mathrm{eV}$ and $T=20 \mathrm{~K}$, (b) $U=0.68 \mathrm{eV}$ and $T=350 \mathrm{~K}$, and (c) $U=0.88 \mathrm{eV}$ and $T=980 \mathrm{~K}$.



(a)

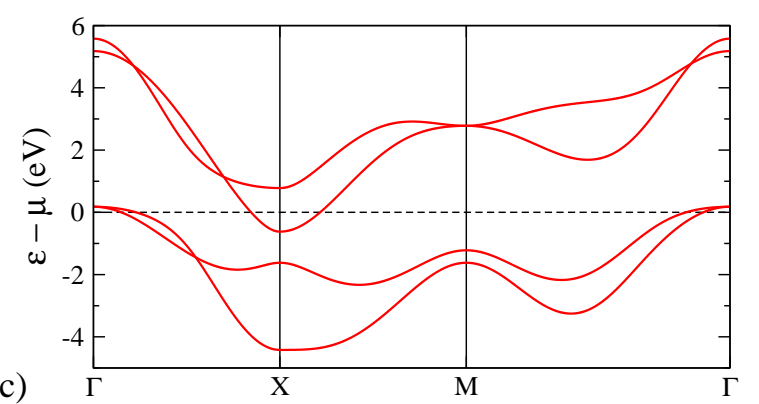

FIG. 7: (a) The Fermi surface of the four-orbital model of Yu et al $\stackrel{54}{5}$ showing the dominant orbital contribution. (b) The orbital weight around the labeled Fermi surfaces in (a). (c) The band structure along high-symmetry directions.

but the sum of the $x z-y z$ and $x z-x y$ terms is comparable. This is to be expected, as the $x z$ orbital dominates the electron pocket at $\mathbf{k}=(\pi, 0)$ [see figure 7]; furthermore, the elliptical form of the electron pocket means that it is nested with the $x z$-dominated regions of the hole pockets, hence accounting for the dominance of $\chi_{1,1}^{-+}$. There is nevertheless significant $y z$ and $x z$ orbital weight around the hole and electron pockets, respectively, accounting for the importance of the inter-orbital susceptibilities $\chi_{1,2}^{-+}$ and $\chi_{1,3}^{-+}$.

Consistent with the mean-field phase diagram, a broad

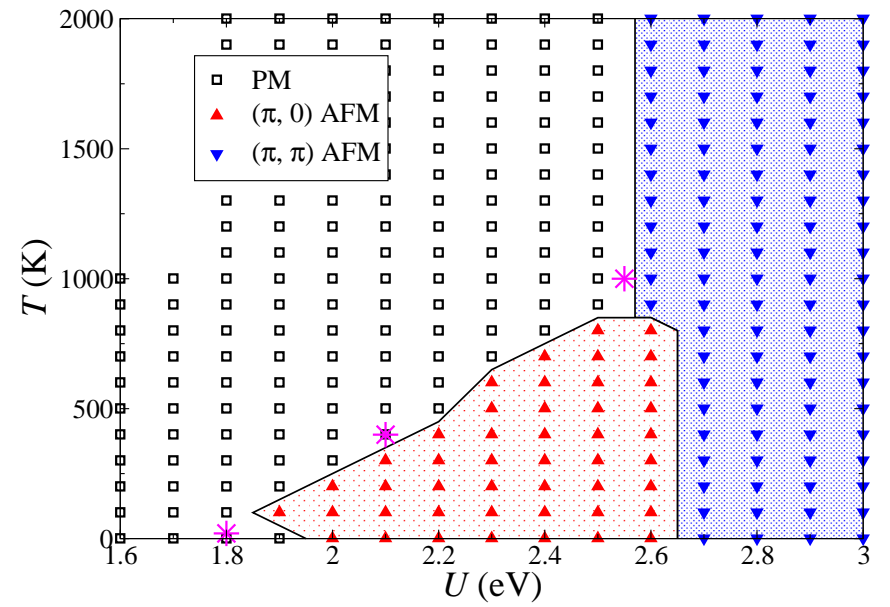

FIG. 8: The $T-U$ mean-field phase diagram of the four-orbital model of $\mathrm{Yu}$ et al. ${ }^{54}$ The lightly-shaded region approximately indicates the extent of the $(\pi, 0)$ AFM phase, while the darklyshaded region gives the extent of the $(\pi, \pi)$ AFM phase. We show the static susceptibility at the points indicated by the star symbols in figure 9

peak develops in $\chi^{-+}(\mathbf{q}, \omega=0)$ at $\mathbf{q}=(\pi, \pi)$ with increasing $U$; close to the $(\pi, \pi)$ AFM state it dominates the magnetic response, see figure 9(c). Although there is low-energy scattering between the two electron pockets with this wavevector, the large magnitudes of the mean-field staggered magnetic potentials $\eta_{\nu}[(6)]$ suggests that the $(\pi, \pi)$ AFM state cannot be understood only in terms of Fermi surface physics. For example, at $U=2.7 \mathrm{eV}$ and $3 \mathrm{eV}$ we have $0.53 \mathrm{eV} \geq\left|\eta_{\nu}\right| \geq 0.43 \mathrm{eV}$ and $1.23 \mathrm{eV} \geq\left|\eta_{\nu}\right| \geq 0.93 \mathrm{eV}$, respectively. The former is comparable to the minimum energy (at the $\mathrm{X}$ points) of the band lying entirely above the Fermi surface; the latter is close to the difference between the hole bands and the Fermi energy at the M point. Since the $\eta_{\nu}$ are the characteristic energies for the reconstruction of the electronic structure in the AFM state, it is therefore likely that these regions participate in the $(\pi, \pi)$ AFM state, 

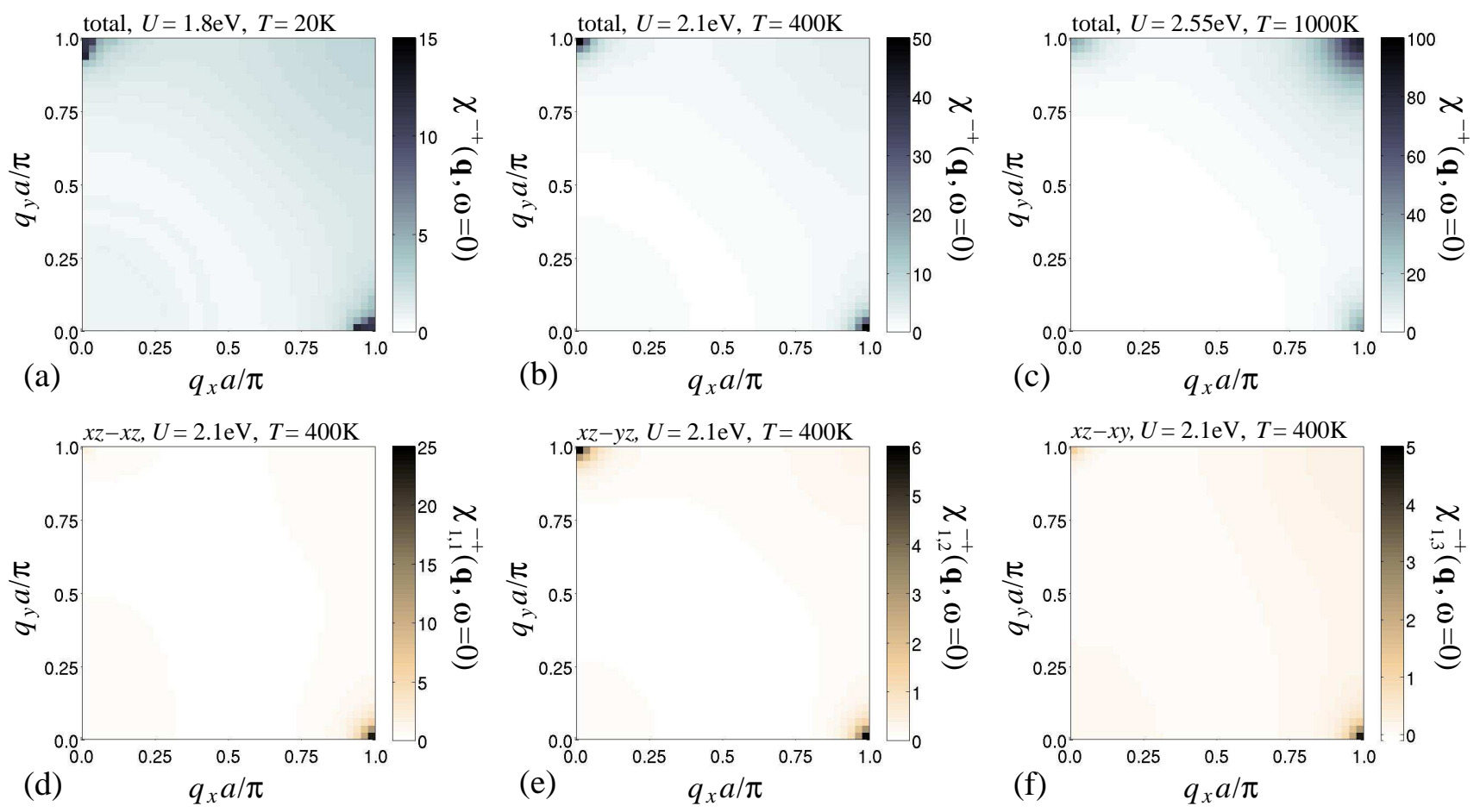

FIG. 9: The static transverse spin susceptibility of the four-orbital model of Yu et al. ${ }^{54}$ at (a) $U=1.8 \mathrm{eV}$ and $T=20 \mathrm{~K}$, (b) $U=2.1 \mathrm{eV}$ and $T=400 \mathrm{~K}$, and (c) $U=2.55 \mathrm{eV}$ and $T=1000 \mathrm{~K}$. The remaining three figures show the dominant contributions to the peak at $\mathbf{q}=(\pi, 0)$ at $U=2.1 \mathrm{eV}$ and $T=400 \mathrm{~K}$ : (d) the $x z-x z$ term, (e) the $x z-x y$ term, and (f) the $x z-x y$ term.

accounting for its much lower free energy compared to the $(\pi, 0)$ AFM phase. .54

\section{The five-orbital model of Kuroki et al.}

The five-orbital model of Kuroki et al $\stackrel{40}{[0}$ includes all five Fe $d$-orbitals. It was constructed by fitting to $a b$ initio calculations for $\mathrm{LaFeAsO}$, and it was one of the first orbital-based models proposed for the iron pnictides. The behaviour of this model, and several of its close relatives,


netic properties are particularly well understood, with RPA calculations confirming that $(\pi, 0)$ AFM order is realized in the undoped model $\frac{4957}{57}$ The extent of this state in the phase diagram at zero doping, and the possible competition with other magnetic phases, nevertheless remains largely unexplored.

This model was originally constructed in an orbital basis where the $x$ and $y$ axes are rotated by $45^{\circ}$ to the $\mathrm{Fe}$ lattice. In the other models considered here, however, the Fe lattice defines the coordinate axes for the orbital basis. To allow direct comparison with these other models, we therefore show the Fermi surface of the five-orbital model of Kuroki et al. in the usual basis in figure 10(a). Although the Fermi surface has the same basic form and orbital composition as the three-orbital model, the electron pockets are almost circular, and there is an additional $x y$-dominated hole pocket at the M point. Furthermore, from the band structure figure 10(c) we see that the hole pockets at the $\Gamma$ point have similar Fermi velocities to the electron pockets, which themselves have much higher Fermi velocity along the $\Gamma-\mathrm{X}$ line than in the $\mathrm{X}-\mathrm{M}$ direction. The latter accounts for the anisotropic spin-wave dispersion found in Ref. 57 .

In constructing our mean-field phase diagram figure 11 we extended the ansatz (3) to also compare the free energy of the ordered states within the rotated orbital basis used by Kuroki et al. to those in the usual orbital basis. We find that the mean-field solution in the usual orbital basis always has the same [PM and $(\pi, \pi)$ AFM] or slightly lower $[(\pi, 0) \mathrm{AFM}]$ free energy than the meanfield solution in the rotated basis. Although our results are not directly comparable with those of Kaneshita et al. ${ }^{47}$ who used the rotated orbital basis and allowed for inter-orbital mean fields, our phase diagram is nevertheless in good qualitative agreement $\underline{47 / 49}$ for $U_{c}>0.85 \mathrm{eV}$ we find a $(\pi, 0)$ AFM state whose critical temperature rapidly grows with increasing $U$. At $U \gtrsim 1.15 \mathrm{eV}$ and $T>1000 \mathrm{~K}$, however, the $(\pi, \pi)$ AFM state is stable.

In figure 12 we present the results for the static transverse susceptibility at the points marked in figure 11 . For $U \lesssim 1 \mathrm{eV}$, the results are rather similar to those already seen in the two- and four-orbital models. In the low-temperature PM state [figure 12(a)], the susceptibility takes a maximum in an arc of radius $\approx 0.05 \pi$ about 

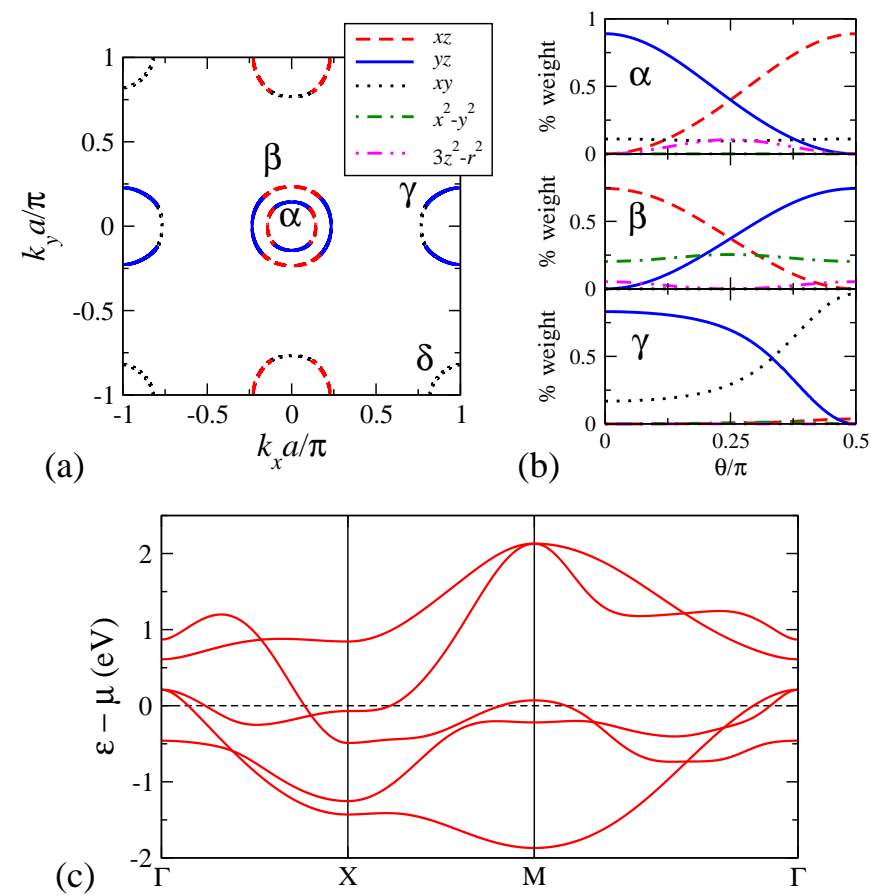

FIG. 10: (a) The Fermi surface of the five-orbital model of Kuroki et al ${ }^{40}$ showing the dominant orbital contribution. (b) The orbital weight around the $\alpha, \beta$ and $\gamma$ Fermi surfaces in (a); the orbital weight around the $\delta$ Fermi surface is not shown as it has almost complete $x y$ character. (c) The band structure along high-symmetry directions.

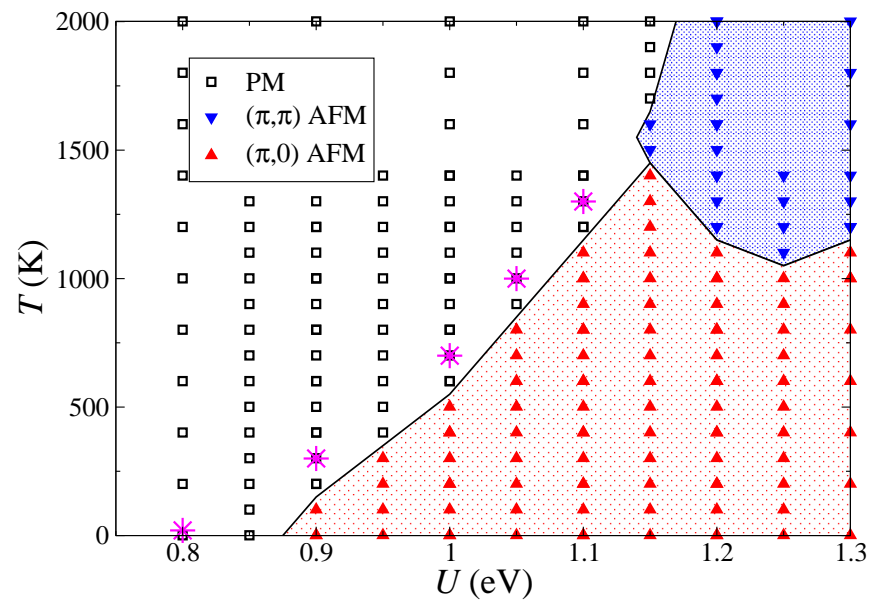

FIG. 11: The $T-U$ mean-field phase diagram of the fiveorbital model of Kuroki et al. ${ }^{40}$ The lightly-shaded region approximately indicates the extent of the $(\pi, 0)$ AFM phase, while the darkly-shaded region gives the extent of the $(\pi, \pi)$ AFM phase. We show the static susceptibility at the points indicated by the star symbols in figure 12 $(\pi, 0)$; at slightly higher $U=0.85 \mathrm{eV}$ we find evidence of an incommensurate AFM state with ordering vector $(0.925 \pi, 0.025 \pi)$. For the points lying directly above the mean-field $(\pi, 0)$ AFM state, however, the susceptibility takes a maximum exactly at $\mathbf{q}=(\pi, 0)$. This peak is nevertheless much broader than in the two- or fourorbital models, indicating a larger distribution of nesting vectors.

The dominant contributions to the peak at $\mathbf{q}=(\pi, 0)$ in the $U=0.9 \mathrm{eV}, T=300 \mathrm{~K}$ static susceptibility [figure 12(b)] are shown in figure 13 . The most important terms are the $y z-y z$ [figure 13(a)], $y z-x y$, [figure 13(b)] and $x y$ - $x y$ [figure [13(c)] susceptibilities, which together constitute $\approx 50 \%$ of the peak height in the total susceptibility; for comparison, in the four-orbital model the dominant susceptibilities shown in figure 9(d-f) are responsible for $\approx 90 \%$ of the $(\pi, 0)$ peak. The dominant terms in Kuroki et al.'s five-orbital model are consistent with nesting of the outer hole pocket with the electron pockets being the main driver of the magnetism, but with the nesting of the hole pocket at the M point with the electron pockets also playing a substantial role. This is supported by the reconstructed Fermi surface shown in Ref. 47 for the low-moment case.

As $U$ is increased to $1.05 \mathrm{eV}$ [figure 12 (d)] the nesting picture of the magnetism begins to break down, with the appearance of a definite (but still subdominant) peak at $\mathbf{q}=(\pi, 0.5 \pi)$. This feature completely dominates the response at $U=1.1 \mathrm{eV}$, indicating that the mean-field phase diagram is unreliable for higher coupling strengths. Peaks at these wavevectors have previously been observed, 5156 where they were explained as due to scattering between the electron Fermi pockets. This appears to be inconsistent with the observation that these peaks are most pronounced at high temperatures $\gtrsim 1000 \mathrm{~K}$, which instead suggests that band structure features away from the Fermi surface are responsible. In particular, we note that there is a region of flat bands at $-0.2 \mathrm{eV}$ near the $\mathrm{M}$ point, which is predominantly derived from the almostfilled $3 z^{2}-r^{2}$ orbital ${ }^{46|51| 60}$ These states are shifted to higher energies by the Hartree terms, and hence might play a major role in the magnetic response at high temperatures. This will be discussed in detail in section IVB.

\section{E. The five orbital model of Graser et al.}

The five-orbital model of Graser et al ${ }^{48}$ is a frequently studied alternative to Kuroki et al.'s model. Graser et al.'s model was also constructed by fitting to $a b$ initio results; as can be seen from the electronic structure shown in figure 14 both five-orbital models share many similarities. The key differences in the Fermi surface are that the electron pockets in this model are clearly elliptical, while the hole pocket at the $\mathrm{M}$ point is almost vanishingly small ${ }^{87}$ The former is in much better agreement with current ARPES and $a b$ initio results; the latter refinement is also in reasonable agreement with $a b i n i$ - 



FIG. 12: The total static transverse spin susceptibility of the five-orbital model of Kuroki et al. ${ }^{40}$ at $($ a) $U=0.8 \mathrm{eV}$ and $T=20 \mathrm{~K}$, (b) $U=0.9 \mathrm{eV}$ and $T=300 \mathrm{~K},(\mathrm{c}) U=1.0 \mathrm{eV}$ and $T=700 \mathrm{~K}$, (d) $U=1.05 \mathrm{eV}$ and $T=1000 \mathrm{~K}$, and (e) $U=1.1 \mathrm{eV}$ and $T=1300 \mathrm{~K}$.


FIG. 13: The dominant contributions to the $(\pi, 0)$-peak at $U=0.9 \mathrm{eV}$ and $T=300 \mathrm{~K}$ in the five-orbital model of Kuroki et al. 40 the (a) $y z-y z$, (b) $y z-x y$, and (c) $x y$ - $x y$ susceptibilities.

tio calculations, but the number of hole pockets remains somewhat unclear in ARPES.13/27/71/72/88 The flat band region near the $\mathrm{M}$ point is also closer to the Fermi surface than in Kuroki et al.'s model; as such, we might expect it to play a larger role in the magnetic response.

The magnetic properties of Graser et al.'s five-orbital model has only recently attracted attention. In Ref. 61 it was shown that the $(\pi, 0)$ AFM state was realized at $T=0 \mathrm{~K}$ for $U \gtrsim 1.23 \mathrm{eV}$, but the authors did not consider competition with other magnetic states. The existence of a $(\pi, 0)$ AFM ground state with realistic ordered moment and Fermi surface was confirmed in Ref. 64 for a rather narrow range of $U$. For much larger values of $U$ it has been shown that Hartree-Fock and Gutzwiller theories give quite different results. ${ }^{68}$ In figure 15 we show the phase diagram in the $T-U$ plane: a $(\pi, 0)$ AFM state with low critical temperature $<500 \mathrm{~K}$ is realized for $1.25 \mathrm{eV} \lesssim$ $U \lesssim 1.35 \mathrm{eV}$, but the phase diagram is clearly dominated by a $(\pi, \pi)$ AFM state with high critical temperature $>2000 \mathrm{~K}$. For most of the $U$ range where the $(\pi, 0) \mathrm{AFM}$ state is stable at $T=0 \mathrm{~K}$, we find that the $(\pi, \pi)$ AFM state is in fact realized at higher $T$, with the PM phase separating them at lower values of $U$.

The results for the static susceptibility [figure 16] in- 

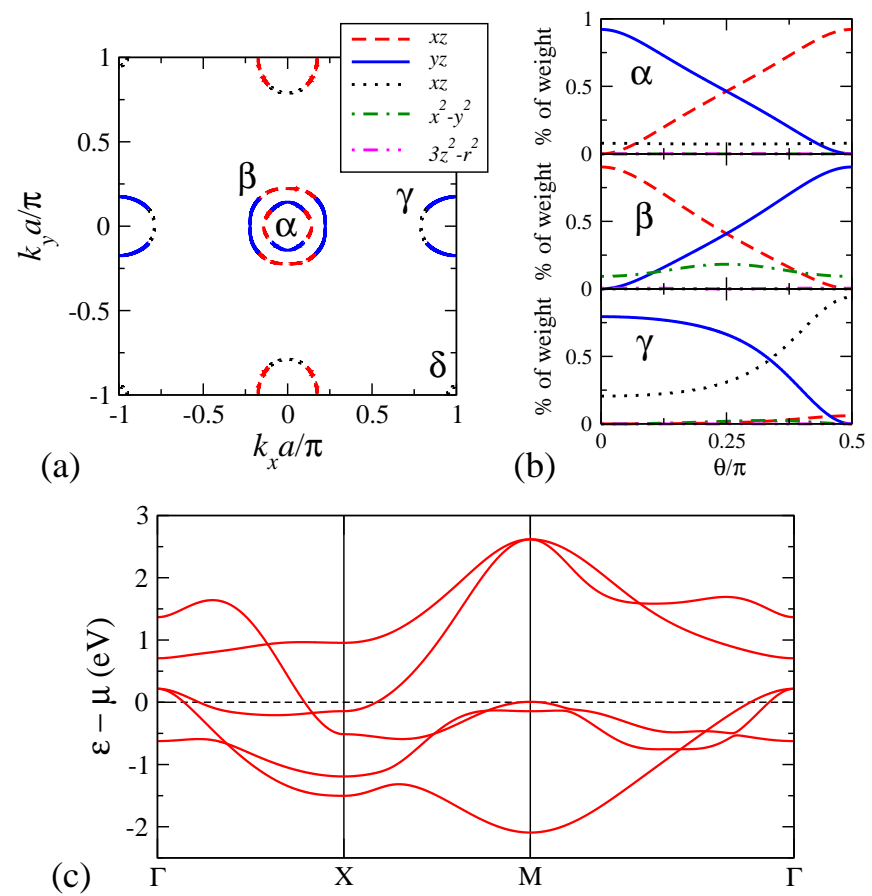

FIG. 14: (a) The Fermi surface of the five-orbital model of Graser et al. ${ }^{48}$ showing the dominant orbital contribution. (b) The orbital weight around the $\alpha, \beta$ and $\gamma$ Fermi surfaces in (a); the orbital weight around the $\delta$ Fermi surface is not shown as it has almost complete $x y$ character. (c) The band structure along high-symmetry directions.

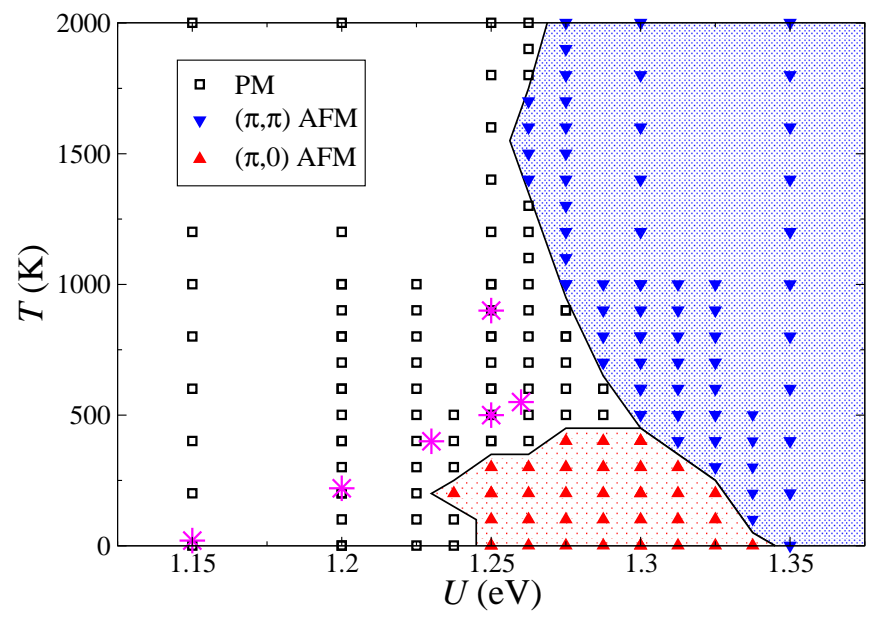

FIG. 15: The $T-U$ mean-field phase diagram of the five-


approximately indicates the extent of the $(\pi, 0)$ AFM phase, while the darkly-shaded region gives the extent of the $(\pi, \pi)$ AFM phase. We show the static susceptibility at the points indicated by the star symbols in figure 16 dicate that the mean-field phase diagram is only partially correct. The evolution of $\chi^{-+}(\mathbf{q}, \omega=0)$ up to the edge of the $(\pi, 0)$ AFM phase is similar to that in Kuroki et al.'s model: as $U$ is increased, a definite tendency to weakly-incommensurate order [with a peak at $\mathbf{q} \approx(0.95 \pi, 0.025 \pi)$ ] gives way to broad peaks at $\mathbf{q}=(\pi, 0)$. We have confirmed an instability towards incommensurate order below at least $100 \mathrm{~K}$ at $U=1.2 \mathrm{eV}$. We also note a ring-like feature centred at $\mathbf{q}=(\pi, \pi)$ in figure 16(a); by $U=1.23 \mathrm{eV}$ this has apparently evolved into a broad peak at $\mathbf{q}=(\pi, 0.6 \pi)$. Although this is similar to the additional peaks seen in the hightemperature results for Kuroki et al.'s model, they appear here at much lower temperatures.

The peak at $(\pi, 0.6 \pi)$ displays a peculiar temperaturedependence. To see this, in figure 16(d) and (e) we show the static susceptibility at $U=1.25 \mathrm{eV}$ for $T=500 \mathrm{~K}$ and $900 \mathrm{~K}$, respectively. In the former case, the peaks at both $(\pi, 0)$ and $(\pi, 0.6 \pi)$ are distinctly visible. We have verified that as the temperature is lowered to $\sim 450 \mathrm{~K}$ the $(\pi, 0)$ peak diverges while the $(\pi, 0.6 \pi)$ peak is somewhat suppressed, and so the $(\pi, 0)$ AFM state is realized at low temperatures in agreement with the mean-field phase diagram. As the temperature is raised to $T=900 \mathrm{~K}$, however, the peak at $(\pi, 0)$ disappears while the $(\pi, 0.6 \pi)$ peak almost doubles in height, revealing that the system is close to an instability towards a $(\pi, 0.6 \pi)$ AFM state. This also suggests that the mechanism responsible for the $(\pi, 0.6 \pi)$ peak is not due to scattering between different Fermi pockets, but rather due to the electronic structure away from the Fermi surface. In figure 16(f) we show the static susceptibility at slightly higher $U$ and $T$ compared to figure $16(\mathrm{~d})$ : the $(\pi, 0.6 \pi)$ peak completely dominates the response, and the system is again close to ordering at this wavevector. Along with figure 16(e), this suggests that the $(\pi, \pi)$ AFM state in figure 15 should be replaced by a $(\pi, 0.6 \pi)$ AFM state; the region of stable $(\pi, 0)$ AFM order will also likely shrink.

To investigate the origin of the $(\pi, 0.6 \pi)$ peak we have examined the dominant contributions to $\chi^{-+}$. Focusing upon the $U=1.25 \mathrm{eV}, T=500 \mathrm{~K}$ case, we find that the susceptibilities involving the $x y$-orbital are most important, contributing $\approx 50 \%$ of the peak height; the largest contributions come from the $x y$ - $x y$ term [figure 17(a)], the $y z$ - $x y$ term [figure $17(\mathrm{~b})$ ], the $x z$ - $x y$ term [figure 17. (b) with $q_{x} \leftrightarrow q_{y}$ ], and the $\left(x^{2}-y^{2}\right)$-xy term [figure 17(c)]. The dominant role of the $x y$ orbital is consistent with the results of Ref. 51 and Ref. 56 for similar orbital models, but it is difficult to reconcile the temperaturedependence observed here with the author's interpretation that the $(\pi, 0.6 \pi)$ peak originates from scattering between the electron pockets. An alternative possibility is scattering between the hole pockets at the $\Gamma$ point and the $x y$-derived hole pocket at the $\mathrm{M}$ point, although the required wavevector is slightly too large. We will discuss this matter further in section IVB 

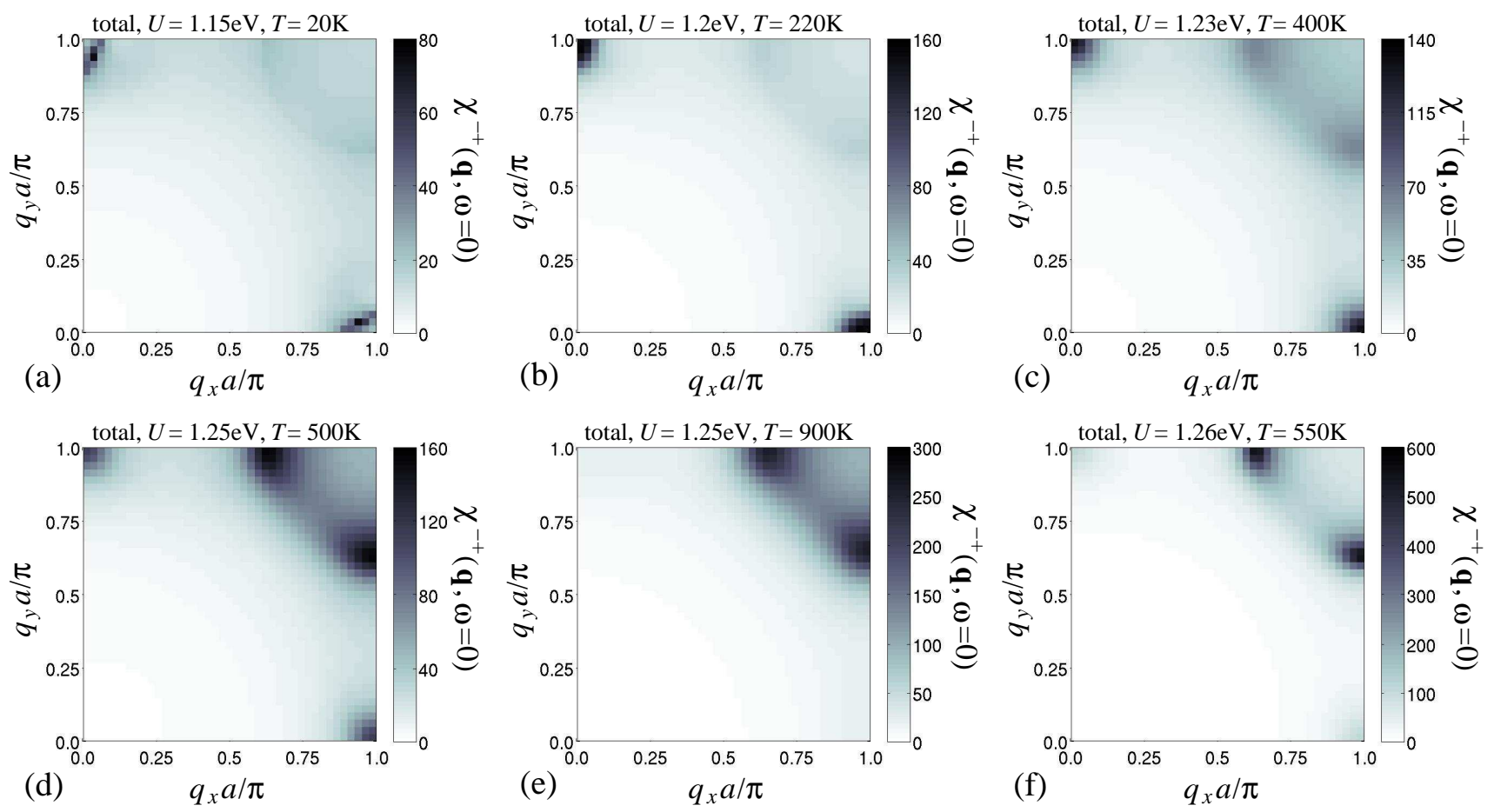

FIG. 16: The total static transverse spin susceptibility of the five-orbital model of Graser et al $\stackrel{48}{a}$ at (a) $U=1.15 \mathrm{eV}$ and $T=20 \mathrm{~K}$, (b) $U=1.2 \mathrm{eV}$ and $T=220 \mathrm{~K}$, (c) $U=1.23 \mathrm{eV}$ and $T=400 \mathrm{~K}$, (d) $U=1.25 \mathrm{eV}$ and $T=500 \mathrm{~K}$, (e) $U=1.25 \mathrm{eV}$ and $T=900 \mathrm{~K}$, and (f) $U=1.26 \mathrm{eV}$ and $T=550 \mathrm{~K}$.
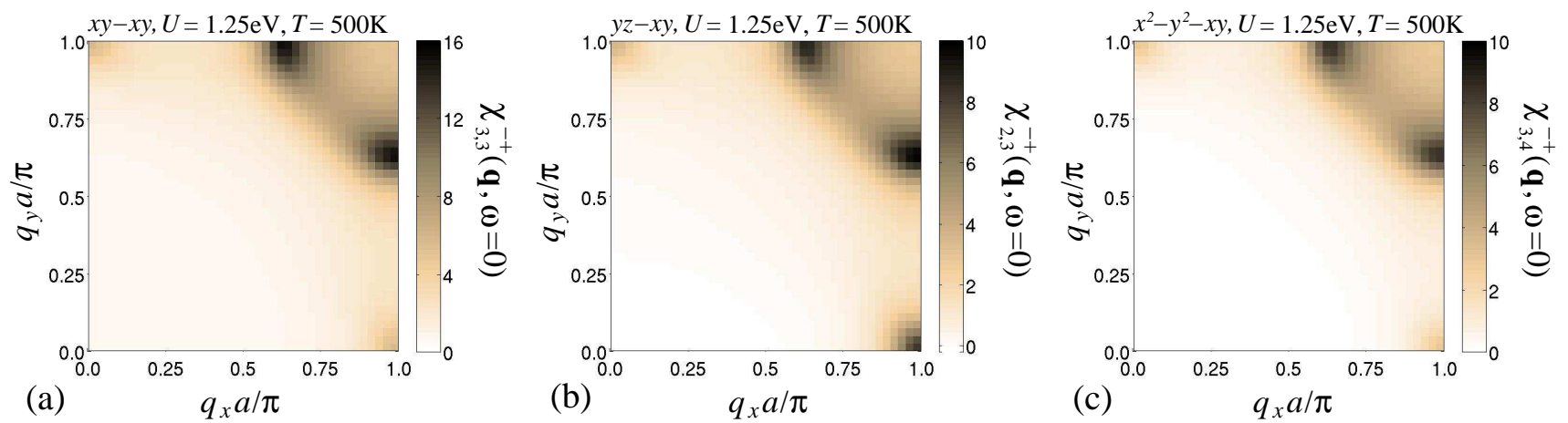

FIG. 17: The dominant contributions to the static transverse spin susceptibility at $U=1.25 \mathrm{eV}, T=500 \mathrm{~K}$ in the five-orbital

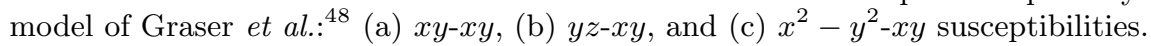

\section{DISCUSSION}

To summarize our main results, we found that the two-

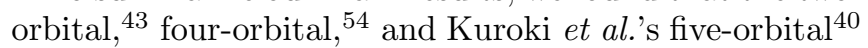
models display a rather robust $(\pi, 0)$ AFM phase in their zero-doping phase diagrams. In both the four- and fiveorbital models, this AFM state competes with a strongcoupling AFM state with different ordering vector. In contrast, the three-orbital ${ }^{59}$ and Graser et al.'s fiveorbita $\sqrt{48}$ models show at most a weak tendency towards $(\pi, 0)$ AFM order: in the former it is likely not present anywhere in the considered phase diagram, while in the latter it is out-competed over much of the phase diagram by a much more stable $(\pi, 0.6 \pi)$ AFM state.

Our results support the scenario of the $(\pi, 0)$ AFM state originating from nesting of the electron and hole Fermi pockets. This is a crucial ingredient for the magnetism: as discussed below in section IVA, the stability of the $(\pi, 0)$ AFM state is positively correlated with the degree of the nesting. The similarity of the electronic structure of the two five-orbital models, yet the very large differences in their magnetic phase diagrams, is also of special note. In particular, this sheds light on the origin of the $(\pi, 0.5 \pi)-(\pi, 0.6 \pi)$ AFM state realized 
in these models at strong coupling or high temperatures. In section IVB we argue that subtle differences in the states derived from the $3 z^{2}-r^{2}$ orbital play a significant role in stabilizing this state. Finally, in section IV C we discuss the implications of our results for models of the magnetic order in the iron pnictides.

\section{A. Nesting}

The $(\pi, 0)$ AFM order was found to be most robust in models where the quality of the nesting between the electron and hole pockets is high. For perfect nesting the dispersion $\epsilon_{\mathbf{k}}$ satisfies

$$
\epsilon_{\mathbf{k}}=-\epsilon_{\mathbf{k}+\mathbf{Q}}
$$

for some nesting vector $\mathbf{Q}$. In such a scenario, not only do the Fermi surfaces exactly overlap upon performing the shift by $\mathbf{Q}$, but also the holes and electrons have the same effective mass. In realistic models such as those considered here the condition (17) is only approximately fulfilled. For a nesting picture to still be relevant, we require that some segments of the electron and hole Fermi surfaces should overlap, and that the effective mass difference between them should not be too great. We note that it is sometimes the case that while the condition (17) is satisfied approximately for the entire Fermi surface for the vector $\mathbf{Q}$, a better match between some Fermi surface segments (but worse for others) may be achieved for a slightly different vector $\widetilde{\mathbf{Q}} \approx \mathbf{Q}$. At weak coupling strengths, where the effective staggered magnetic potential is small, more of the Fermi surface may be gapped at ordering vector $\widetilde{\mathbf{Q}}$ than at $\mathbf{Q}$.

In figure 18, we show the Fermi surface of each of the models superimposed with the Fermi surface translated by $\mathbf{Q}=(\pi, 0)$. It is immediately clear that the threeorbital model has the worst nesting properties: not only is there very poor overlap between the hole Fermi surface and the translated electron Fermi surface, but also the electron pocket has much lower effective mass than either of the hole pockets. It is therefore not surprising that this is the only model that fails to show the required $(\pi, 0)$ AFM order.

Although both electron pockets participate in the $(\pi, 0)$ AFM state in the two-orbital model, the nesting here is nevertheless rather similar to that in the fourorbital model. In these two models, a segment near the minor axis of the shifted elliptical electron pocket overlaps with the small hole pocket, while a segment near the major axis overlaps with the large hole pocket [see figure $18(\mathrm{a})$ and (c)]. There is an excellent match between the dominant orbital character of the original and the shifted Fermi surfaces, which should enhance the AFM fluctuations as the intra-orbital Coulomb repulsion is much larger than the inter-orbital Hund's rule coupling.

The nesting of the electron pockets with the outer hole pocket at the $\Gamma$ point in Kuroki et al.'s five-orbital model is the best considered here, with almost complete overlap with the shifted Fermi surfaces. The orbital-resolved susceptibilities suggests that this is most important to the magnetic instability, although the poorer nesting of the electron pockets with the hole pocket at the $\mathrm{M}$ point is likely responsible for broadening the peak at $(\pi, 0)$. Unlike the two- and four-orbital models, therefore, there is a relatively poor match of the dominant orbital character of the nested Fermi surfaces. This is reflected in the susceptibilities: $\sum_{\nu} \chi_{\nu, \nu}^{-+}$contributes $\approx 30 \%$ of the height of the $(\pi, 0)$ peak in Kuroki et al.'s model, while in the fourorbital model the susceptibility $\chi_{1,1}^{-+}$is alone responsible for $\approx 50 \%$ of the peak height. In contrast, despite the near-identical orbital content of the Fermi surfaces, the instability towards $(\pi, 0)$ AFM order in the five-orbital model of Graser et al. is much weaker. This can be explained by the absence of direct Fermi-surface overlap between the electron and the hole pockets at the $\Gamma$ point, and also the much weaker nesting between electron pockets and the small hole pocket at the M point. The stark differences between the two five-orbital models highlights the sensitivity of the $(\pi, 0)$ AFM state to subtle details of the electronic structure.

Our results suggest that a qualitatively-correct understanding of the AFM order in the pnictides can be developed without reference to the orbital structure, i.e. they support the excitonic theories of the magnetism. This is not unexpected, as the $(\pi, 0)$ AFM order is realized at relatively weak coupling strengths, where the excitonic model arises naturally as a low-energy effective theory ${ }^{29 \mid 33}$ The rather strong competition with other AFM states, however, is not anticipated by such excitonic models.

\section{B. Hartree shifts}

The inclusion of Hartree shifts in our mean-field decoupling scheme requires justification: models where the tight-binding band structure is constructed by fitting to $a b$ initio results should already account for the Hartree shifts, so it is then only necessary to include the staggered magnetic potentials in (4). It is nevertheless likely that such methods underestimate the effect of the correlations; indeed, comparison with ARPES reveals that the bands obtained within the local density approximation are subject to significant energy shifts 12 Including the Hartree shifts is the simplest way to test the robustness of the AFM order towards such modifications of the band structure. In Ref. 56 it was reported that the Hartree shifts can lead to dramatic changes in the Fermi surface of Kuroki et al.'s five-orbital model. Ikeda and co-workers similarly found unphysical renormalization of the Fermi surface by the $\omega=0$ self-energy corrections calculated within the fluctuation-exchange approximation. $46 \mid 5160$

In order to evaluate the effect of the Hartree shifts, we have calculated the renormalized band structures for each model in a fictitious $T=0 \mathrm{~K}$ PM state at a coupling 



(e)

FIG. 18: Nesting properties of the orbital models. We show the Fermi surface (thick lines) and the Fermi surface translated

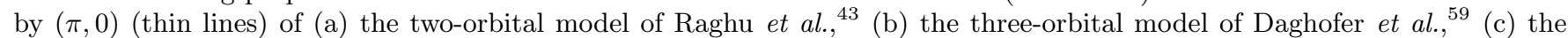

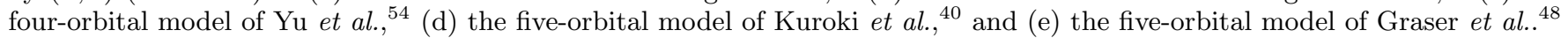

strength where an AFM mean-field state is realized. This is a good estimate of the band structure in the stable $T>$ $0 \mathrm{~K}$ PM state as there is only rather small temperaturedependence of the orbital occupancies. The degeneracy of the $x z$ and $y z$ orbitals in the two-orbital model means that there is no change in the band structure; for the three- and four-orbital models we find only very slight changes in the band structure far from the Fermi surface ( $\gtrsim 0.5 \mathrm{eV}$ and $1 \mathrm{eV}$, respectively). As such, the inclusion of the Hartree shifts should be irrelevant to the weakcoupling magnetic response of these models.

For the five-orbital models we again find very minor changes in the Fermi surface, limited to the shrinking (Kuroki et al.) or removal (Graser et al.) of the $x y$ derived hole pocket at the $M$ point, which is due to the positive Hartree shift of the other orbitals relative to the (least-occupied) $x y$ orbital. As shown in figure 19(a) and (c), however, the flat-band region near the $M$ point and below the Fermi energy undergoes an almost uniform shift to higher energies. These states are mostly derived from the $3 z^{2}-r^{2}$ orbital, which has the highest occupation and hence has the largest Hartree shift $46|48| 51 / 60]$ The shift to higher energies is particu- larly dramatic in Graser et al.'s model, where the flat band region is shifted from $\approx-0.15 \mathrm{eV}$ to $\approx-0.07 \mathrm{eV}$; in Kuroki et al.'s model the equivalent feature is shifted from $\approx-0.21 \mathrm{eV}$ to $\approx-0.17 \mathrm{eV}$. We note that the Hartree shifts of the orbitals are in qualitative agreement with the more advanced analysis of Ikeda et al. ${ }^{46|51| 60}$ although the change in the band structure is less severe in our results.

The shift of the $\left(3 z^{2}-r^{2}\right)$-derived states closer to the Fermi surface suggests that they might play a role in the high- $T$ and $U$ magnetic behaviour of the system, where the $(\pi, 0)$ AFM state competes with an enigmatic $(\pi, 0.5 \pi)-(\pi, 0.6 \pi)$ AFM state. This is contradicted by the result from section IIIE that the peak at $(\pi, 0.6 \pi)$ is mostly due to the $x y$ orbital, with the $3 z^{2}-r^{2}$ orbital making a relatively modest contribution. To isolate the effect of the $3 z^{2}-r^{2}$ orbital, we have therefore recalculated the static susceptibility without including the Hartree shifts, $\tilde{\chi}^{-+}(\mathbf{q}, \omega=0)$. In figure $19(\mathrm{~b})$ and $(\mathrm{d})$ we show the ratio of the static susceptibility with the Hartree shift to that without for the cases figure 12(d) $(U=1.05 \mathrm{eV}, T=1000 \mathrm{~K}$, Kuroki et al.'s model) and figure 16 (d) $(U=1.25 \mathrm{eV}, T=500 \mathrm{~K}$, Graser et al.'s 
Kuroki et al.

(a)



Graser et al.

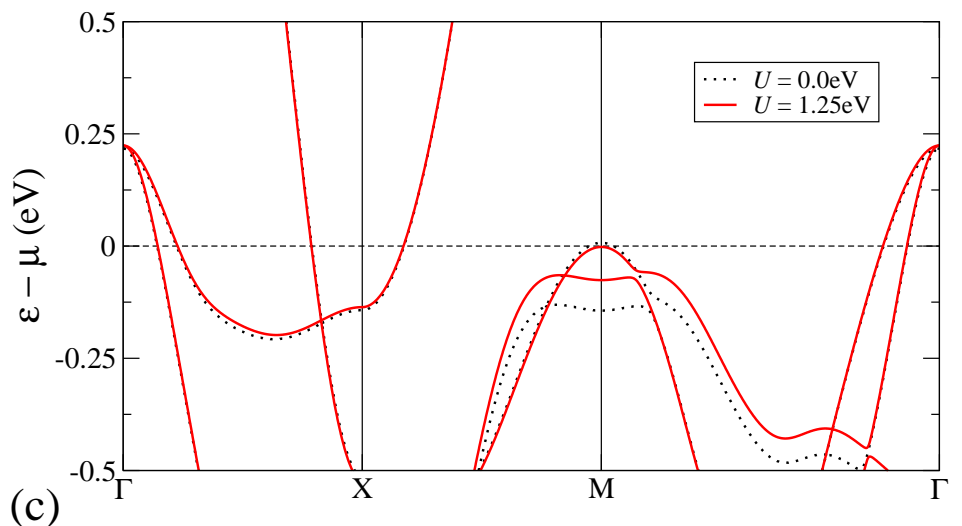

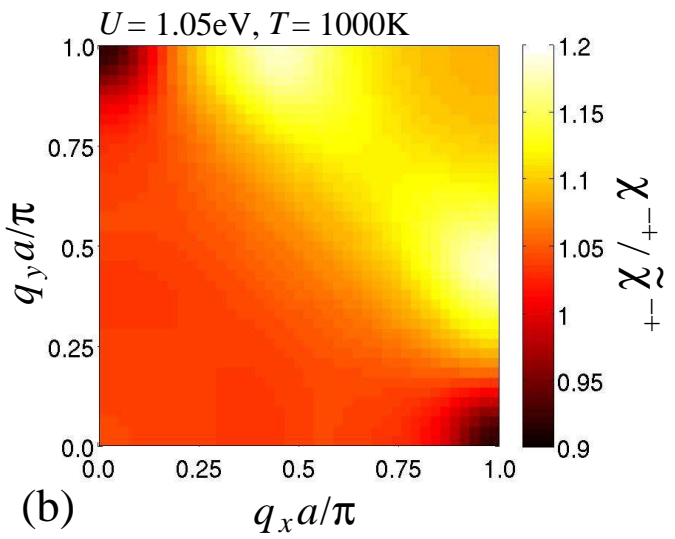



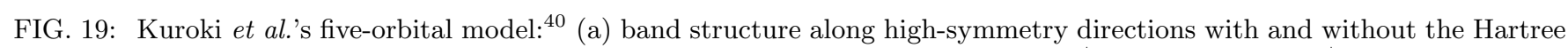
shift at $U=1.05 \mathrm{eV}, T=0 \mathrm{~K}$; (b) ratio of the static transverse susceptibility with $\left(\chi^{-+}\right)$and without $\left(\widetilde{\chi}^{-+}\right)$the Hartree shift

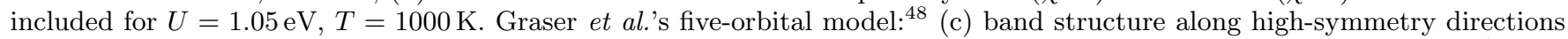
with and without the Hartree shift at $U=1.25 \mathrm{eV}, T=0 \mathrm{~K} ;(\mathrm{d})$ ratio of the static transverse susceptibility with $\left(\chi^{-+}\right)$and without $\left(\widetilde{\chi}^{-+}\right)$the Hartree shift included for $U=1.25 \mathrm{eV}, T=500 \mathrm{~K}$.

model). In both cases, we see that the peak at $(\pi, 0.5 \pi)$ $(\pi, 0.6 \pi)$ is significantly enhanced by the inclusion of the Hartree shifts, especially so for Graser et al.'s model. Including the Hartree shifts also leads to a reduction of the height of the peak at $(\pi, 0)$, probably due to the shrinking of the $x y$ pocket, highlighting the sensitive dependence of the $(\pi, 0)$ AFM state upon fine details of the nesting.

As the rest of the band structure undergoes relatively little change upon the inclusion of the Hartree shifts, the results figure 19 (b) and (d) indicate that the shift of the $3 z^{2}-r^{2}$-derived flat band near the $\mathrm{M}$ point is heavily involved in stabilizing the $(\pi, 0.5 \pi)-(\pi, 0.6 \pi)$ AFM state. This is paradoxical: although the $3 z^{2}-r^{2}$ orbitals are clearly important to the appearance of the $(\pi, 0.5 \pi)$ $(\pi, 0.6 \pi)$ AFM state, other orbitals dominate the magnetic response at the ordering vector. Furthermore, the inclusion of the Hartree shifts enhances all the orbitally resolved susceptibilities $\chi_{\mu, \nu}^{-+}$by a similar factor. For example, in the $U=1.25 \mathrm{eV}, T=500 \mathrm{~K}$ results for Graser et al.'s model, the ratio $2.3 \gtrsim \chi_{\mu, \nu}^{-+} / \widetilde{\chi}_{\mu, \nu}^{-+} \gtrsim 1.8$ for all $\mu$ and $\nu$, although it is maximal when the $3 z^{2}-r^{2}$ orbital is involved. This appears to indicate that the flat band near the $\mathrm{M}$ point plays an indirect role in the magnetic ordering. We speculate that this involves the strong peak in the density of states associated with this band, which feeds into the response of the other bands due to the orbital mixing.

The sensitivity of the $(\pi, 0.5 \pi)-(\pi, 0.6 \pi)$ peak to the Hartree shift implies a strong dependency upon the choice of the ratio $J / U$. For $J / U=0.25$ considered here, we have positive effective Hartree shifts $\widetilde{\epsilon}_{\nu}=$ $0.25 U\left(n_{\nu}-\min \left\{n_{\mu}\right\}\right)$. Had we chosen $J / U=0.2$, however, the Hartree shifts would vanish; for $J / U<0.2$ we find negative Hartree shifts, and the $3 z^{2}-r^{2}$ orbital would therefore be shifted away from the Fermi energy, likely suppressing the $(\pi, 0.5 \pi)-(\pi, 0.6 \pi)$ AFM order. 


\section{Implications for models of the iron pnictides}

One of the aims of this work was to identify an ideal low-energy orbital model of the pnictides, i.e. a model possessing Fermi surfaces in good quantitative agreement with ARPES and $a b$ initio results, and also displaying robust $(\pi, 0)$ order. Unfortunately, none of the models studied here satisfy both criteria. The models which display robust $(\pi, 0)$ AFM order have rather unrealistic elements in their electronic structure: the two-orbital model has a $x z / y z$-derived hole pocket at the $\mathrm{M}$ point and also neglects the large $x y$ weight at the Fermi energy, in the four-orbital model the $x y$ weight is also unrealistically small at the Fermi surface and the electron pockets do not involve a band crossing, while the electron pockets in Kuroki et al.'s five-orbital model are not elliptical. In contrast, the three-orbital model qualitatively captures the predicted Fermi surface topology and dominant orbital contributions, while Graser et al.'s five-orbital model is in good quantitative agreement, but these two models show the weakest tendencies towards $(\pi, 0)$ AFM order.

The sensitivity of the $(\pi, 0)$ AFM state to small details of the band structure raises an important question about the magnetic state in the pnictides: if electron-hole nesting is indeed responsible for this state, why is it realized in such a large range of compounds, each with subtly different Fermi surfaces?60 A possible answer is that the coupling to the lattice degrees of freedom allows the system to fine-tune its Fermi surface. Indeed, the magnetic properties of these compounds have been shown to be strongly coupled to details of the lattice structure: the lattice constants and pnictogen height above the Fe plane obtained in $a b$ initio calculations are strongly affected by the iron moment, $89[90$ and varying the Fe-As-Fe bond angles can significantly alter the Fermi surface. It is certainly suggestive that Kuroki et al.'s model was fitted to $a b$ initio results using experimental crystal parameters, whereas Graser et al.'s is based on structurallyrelaxed calculations. 92

Moreover, a structural phase transition from a high$T$ tetragonal to low- $T$ orthorhombic phase occurs either just above ( $R \mathrm{FeAsO}$ compounds) or at $\left(A \mathrm{Fe}_{2} \mathrm{As}_{2}\right.$ compounds) the AFM transition temperature. ${ }^{3 / 577}$ A scenario for a joint magnetic-structural transition in the pnictides was proposed within the context of a simple excitonic model in Ref. 93 the lattice distortion improves the nesting condition sufficiently to allow the appearance of an AFM state, which in turn stabilizes the distortion. This was verified for a more realistic four-band excitonic model in Ref. 32, Further work to clarify the role of the orthorhombic distortion is clearly necessary. It would be particularly interesting to examine this question within an orbital model, as the directional wave functions of the orbitals naturally couple to the lattice. The possibility that spontaneous orbital ordering drives the structural transition is of particular interest 9495 Of course, another possible explanation for the stability of the $(\pi, 0)$ AFM order involves strong-coupling physics beyond the nesting scenario $\underline{65 \mid 68}$ We note that recent calculations using dynamical mean-field theory suggest that the correlation strength in these materials has been underestimated 96 Further research is clearly needed to understand the complex magnetic physics of the pnictides.

\section{SUMMARY}

In this work we have presented a systematic weakcoupling investigation into the appearance of the expected $(\pi, 0)$ AFM order in five different orbital models of the iron pnictide parent compounds. The mean-field phase diagrams of each model were first determined, and then the static spin susceptibility was calculated within RPA in the PM state close to the boundaries of the magnetic phases. The highest peaks in the spin susceptibility reveal the actual ordering vector of the nearby mean-field state. The origin of these peaks was studied by examining the dominant orbitally resolved contributions to the total spin susceptibility. This procedure allowed an unbiased assessment of the magnetic-ordering properties of each model.

The studied models display an unexpectedly rich range of magnetic behaviour. Only four of the models were found to have an instability to an AFM state with the required ordering vector, although the robustness of this phase varies greatly between them. In each of these models we also uncovered evidence of a weaklyincommensurate AFM phase with ordering vector close to $(\pi, 0)$ at coupling strengths below the minimum required for a stable $(\pi, 0)$ state. The $(\pi, 0)$ AFM state originates from the nesting of the electron and hole Fermi pockets. The quality of the nesting appears to be of primary relevance for the stability of the $(\pi, 0)$ AFM order, while the matching of the orbital character of the nested Fermi surfaces is of lesser importance. In the most realistic five-orbital models the $(\pi, 0)$ AFM state was found to compete with a strong-coupling incommensurate magnetic state. We have argued that the origin of this phase involves electronic structure below the Fermi energy. The implications of our results for orbital models of the pnictides was discussed, and we propose that the apparent sensitivity of the $(\pi, 0)$ AFM order to fine details of the low-energy electronic structure indicates that lattice degrees of freedom should be included in a theoretical description of the magnetism of the pnictides.

\section{Acknowledgments}

The authors thank I. Eremin, J. Knolle, K Kuroki, and P. Thalmeier for useful discussions. PMRB and CT acknowledge funding from the DFG priority program 1458 and MD from the Emmy-Noether program of the DFG. 
* Electronic address: brydon@theory.phy.tu-dresden.de

$\dagger$ Electronic address: m.daghofer@ifw-dresden.de

¥ Electronic address: carsten.timm@tu-dresden.de

1 Y. Kamihara, T. Watanabe, M. Hirano, and H. Hosono, J. Am. Chem. Soc. 130, 3296 (2008).

2 M. Rotter, M. Tegel, and D. Johrendt, Phys. Rev. Lett. 101, 107006 (2008).

3 J. Zhao, Q. Huang, C. de la Cruz, S. Li, J. W. Lynn, Y. Chen, M. A. Green, G. F. Chen, G. Li, Z. Li, J. L. Luo, N. L. Wang, P. Dai, Nature Materials 7, 953 (2008); J. Zhao, Q. Huang, C. de la Cruz, J. W. Lynn, M. D. Lumsden, Z. A. Ren, J. Yang, X. Shen, X. Dong, Z. Zhao, P. Dai, Phys. Rev. B 78, 132504 (2008).

4 A. Jesche, N. Caroca-Canales, H. Rosner, H. Borrmann, A. Ormeci, D. Kasinathan, K. Kaneko, H. H. Klauss, H. Luetkens, R. Khasanov, A. Amato, A. Hoser, C. Krellner, and C. Geibel, Phys. Rev. B 78, 180504(R) (2008).

${ }^{5}$ Q. Huang, Y. Qiu, W. Bao, M. A. Green, J. W. Lynn, Y. C. Gasparovic, T. Wu, G. Wu, and X. H. Chen, Phys. Rev. Lett. 101, 257003 (2008).

${ }^{6}$ P. A. Lee, N. Nagaosa, and X.-G. Wen, Rev. Mod. Phys. 78, 17 (2006).

7 M.D. Lumsden and A.D. Christianson, J. Phys.: Condens. Matter 22, 203203 (2010).

8 D. C. Johnston, Adv. Phys. 59, 803 (2010).

9 C. de la Cruz, Q. Huang, J. W. Lynn, J. Li, W. Ratcliff II, J. L. Zarestky, H. A. Mook, G. F. Chen, J. L. Luo, N. L. Wang, and P. Dai, Nature 453, 899 (2008).

10 D. K. Pratt, M. G. Kim, A. Kreyssig, Y. B. Lee, G. S. Tucker, A. Thaler, W. Tian, J. L. Zarestky, S. L. Bud'ko, P. C. Canfield, B. N. Harmon, A. I. Goldman, and R. J. McQueeney, arXiv:1104.0717

11 S. E. Sebastian, J. Gillett, N. Harrison, P. H. C. Lau, C. H. Mielke, and G. G. Lonzarich, J. Phys: Condens. Matter 20, 422203 (2008); J. G. Analytis, R. D. McDonald, J.-H. Chu, S. C. Riggs, A. F. Bangura, C. Kucharczyk, M. Johannes, and I. R. Fisher, Phys. Rev. B 80, 064507 (2009).

12 M. Yi, D. H. Lu, J. G. Analytis, J.-H. Chu, S.-K. Mo, R.-H. He, M. Hashimoto, R. G. Moore, I. I. Mazin, D. J. Singh, Z. Hussain, I. R. Fisher, and Z.-X. Shen, Phys. Rev. B 80, 174510 (2009).

13 T. Shimojima, K. Ishizaka, Y. Ishida, N. Katayama, K. Ohgushi, T. Kiss, M. Okawa, T. Togashi, X.-Y. Wang, C.T. Chen, S. Watanabe, R. Kadota, T. Oguchi, A. Chainani, and S. Shin, Phys. Rev. Lett. 104, 057002 (2010).

14 M. A. McGuire, A. D. Christianson, A. S. Sefat, B. C. Sales, M. D. Lumsden, R. Jin, E. A. Payzant, D. Mandrus, Y. Luan, V. Keppens, V. Varadarajan, J. W. Brill, R. P. Hermann, M. T. Sougrati, F. Grandjean, G. J. Long, Phys. Rev. B 78, 094517 (2008); M. A. McGuire, R. P. Hermann, A. S. Sefat, B. C. Sales, R. Jin, D. Mandrus, F. Grandjean, and G. J. Long, New J. Phys. 11, 025011 (2009).

15 R. H. Liu, G. Wu, T. Wu, D. F. Fang, H. Chen, S. Y. Li, K. Liu, Y. L. Xie, X. F. Wang, R. L. Yang, L. Ding, C. He, D. L. Feng, and X. H. Chen, Phys. Rev. Lett. 101, 087001 (2008).

16 J. K. Dong, L. Ding, H. Wang, X. F. Wang, T. Wu, G. Wu, X. H. Chen, and S. Y. Li, New J. Phys. 10, 123031 (2008).

17 S.-L. Drechsler, H. Rosner, M. Grobosch, G. Behr, F. Roth, G. Fuchs, K. Koepernik, R. Schuster, J. Malek, S. Elgaz- zar, M. Rotter, D. Johrendt, H-H. Klauss, B. Büchner, and M. Knupfer, arXiv:0904.0827.

18 W. L. Yang, P. O. Velasco, J. D. Denlinger, A. P. Sorini, CC. Chen, B. Moritz, W.-S. Lee, F. Vernay, B. Delley, J.-H. Chu, J. G. Analytis, I. R. Fisher, Z. A. Ren, J. Yang, W. Lu, Z. X. Zhao, J. van den Brink, Z. Hussain, Z.-X. Shen, and T. P. Devereaux, Phys. Rev. B 80, 014508 (2009).

19 Despite their similar crystaline and electronic structure, it is likely that the iron chalcogenides are much more strongly correlated and have been suggested as better described by a local moment picture

${ }^{20}$ F. Ma, J. Wei, J. Hu, Z.-Y. Lu, and T. Xiang, Phys. Rev. Lett. 102, 177003 (2009).

21 T. Yildirim, Phys. Rev. Lett. 101, 057010 (2008).

22 G. S. Uhrig, M. Holt, J. Oitmaa, O. Sushkov, and R. P. P. Singh, Phys. Rev. B 79, 092416 (2009).

23 F. Krüger, S. Kumar, J. Zaanen, and J. van den Brink, Phys. Rev. B 79, 054504 (2009).

24 B. Schmidt, M. Siahatgar, and P. Thalmeier, Phys. Rev. B 81, 165101 (2010).

25 J. Zhao, D. T. Adroja, D.-X. Yao, R. Bewley, S. Li, X. F. Wang, G. Wu, X. H. Chen, J. Hu, and P. Dai, Nat. Phys. 5, 555 (2009).

26 D. J. Singh and M.-H. Du, Phys. Rev. Lett. 100, 237003 (2008); I. I. Mazin, D. J. Singh, M. D. Johannes, and M. H. Du, ibid 101, 057003 (2008).

27 Y.-Z. Zhang, I. Opahle, H. O. Jeschke, and R. Valentí, Phys. Rev. B 81, 094505 (2010).

28 M. M. Korshunov and I. Eremin, Europhys. Lett. 83, 67003 (2008); Phys. Rev. B 78, 140509(R) (2008).

29 A. V. Chubukov, D. Efremov, and I. Eremin, Phys. Rev. B 78, 134512 (2008).

${ }^{30}$ Q. Han, Y. Chen, and Z. D. Wang, Europhys. Lett. 82, 37007 (2008).

31 A. B. Vorontsov, M. G. Vavilov, and A. V. Chubukov, Phys. Rev. B 79, 060508(R) (2009).

32 P. M. R. Brydon and C. Timm, Phys. Rev. B 79, 180504(R) (2009).

33 V. Cvetkovic and Z. Tesanovic Europhys. Lett. 85, 37002, (2009); Phys. Rev. B 80, 024512 (2009).

34 P. M. R. Brydon and C. Timm, Phys. Rev. B 80, 174401 (2009).

35 R. Thomale, C. Platt, J. Hu, C. Honerkamp, and B. A. Bernevig, Phys. Rev. B 80, 180505(R) (2009).

36 J. Knolle, I. Eremin, A. V. Chubukov, and R. Moessner, Phys. Rev. B 81, 140506(R) (2010).

37 I. Eremin and A. V. Chubukov, Phys. Rev. B 81, 024511 (2010).

38 J. Knolle, I. Eremin, A. Akbari, and R. Moessner, Phys. Rev. Lett. 104, 257001 (2010).

39 R. M. Fernandes and J. Schmalian, Phys. Rev. B 82, 014521 (2010).

${ }^{40}$ K. Kuroki, S. Onari, R. Arita, H. Usui, Y. Tanaka, H. Kontani, and H. Aoki, Phys. Rev. Lett. 101, 087004 (2008).

41 J. Lorenzana, G. Seibold, C. Ortix, and M. Grilli, Phys. Rev. Lett. 101, 186402 (2008).

42 M. Daghofer, A. Moreo, J. A. Riera, E. Arrigoni, D. J. Scalapino, and E. Dagotto, Phys. Rev. Lett. 101, 237004 (2008).

43 S. Raghu, Z.-L. Qi, C.-X. Liu, D. J. Scalapino, and S.-C. Zhang, Phys. Rev. B 77, 220503(R) (2008). 
44 P. A. Lee and X.-G. Wen, Phys. Rev. B 78, 144517 (2008).

45 Y. Yanagi, Y. Yamakawa, and Y. Ōno, J. Phys. Soc. Jpn. 77, 123701 (2008).

${ }^{46}$ H. Ikeda, J. Phys. Soc. Jpn. 77, 123707 (2008).

47 E. Kaneshita, T. Morinari, and T. Tohyama, Phys. Rev. Lett. 103, 247202 (2009).

48 S. Graser, T. A. Maier, P. J. Hirschfeld, and D. J. Scalapino, New J. Phys. 11, 025016 (2009).

49 T. Kariyado and M. Ogata, J. Phys. Soc. Jpn. 78, 043708 (2009).

50 K. Kubo and P. Thalmeier, J. Phys. Soc. Jpn. 78, 083704 (2009).

51 R. Arita and H. Ikeda, J. Phys. Soc. Jpn. 78, 113707 (2009).

${ }^{52}$ Y. Ran, F. Wang, H. Zhai, A. Vishwanath, and D.-H. Lee, Phys. Rev. B 79, 014505 (2009).

53 S.-L. Yu, J. Kang, and J.-X. Li, Phys. Rev. B 79, 064517 (2009).

54 R. Yu, K. T. Trinh, A. Moreo, M. Daghofer, J. A. Riera, S. Haas, and E. Dagotto, Phys. Rev. B 79, 104510 (2009).

55 A. Moreo, M. Daghofer, J. A. Riera, and E. Dagotto, Phys. Rev. B 79, 134502 (2009).

${ }^{56}$ K. Kuroki, H. Usui, S. Onari, R. Arita, and H. Aoki, Phys. Rev. B 79, 224511 (2009).

57 E. Kaneshita and T. Tohyama, Phys. Rev. B 82, 094441 (2010).

58 E. Bascones, M. J. Calderón, and B. Valenzuela, Phys. Rev. Lett. 104, 227201 (2010).

59 M. Daghofer, A. Nicholson, A. Moreo, and E. Dagotto, Phys. Rev. B 81, 014511 (2010).

${ }^{60}$ H. Ikeda, R. Arita, and J. Kuneš, Phys. Rev. B 81, 054502 (2010).

61 M. Daghofer, Q.-L. Luo, R. Yu, D. X. Yao, A. Moreo, and E. Dagotto, Phys. Rev. B 81, 180514(R) (2010).

${ }^{62}$ M. S. Long, L. B. Hu, and W. LiMing, Eur. Phys. J. B 75, 205 (2010).

${ }^{63}$ R. Thomale, C. Platt, W. Hanke, and B. A. Bernevig, arXiv:1002.3599

64 Q. Luo, G. Martins, D.-X.Yao, M. Daghofer, R. Yu, A. Moreo, and E. Dagotto, Phys. Rev. B 82, 104508 (2010).

65 S. Zhou and Z. Wang, Phys. Rev. Lett. 105, 096401 (2010).

${ }^{66}$ F. Yang, H. Zhai, F. Wang, and D.-H. Lee, Phys. Rev. B 83, 134502 (2010).

67 K. Kubo and P. Thalmeier, arXiv:1010.4626

68 T. Schickling, F. Gebhard, and J. Bünemann, arXiv:1011.6219

69 L. V. Keldysh and Y. V. Kopaev, Sov. Phys. Solid State 6, 2219 (1965); J. des Cloizeaux, J. Phys. Chem. Solids 26, 259 (1965); D. Jérome, T. M. Rice, and W. Kohn, Phys. Rev. 158, 462 (1967).

70 D. W. Buker, Phys. Rev. B 24, 5713 (1981).

${ }^{71}$ L. Boeri, O. V. Dolgov, and A. A. Golubov, Phys. Rev. Lett. 101, 026403 (2008).

72 H. Eschrig and K. Koepernik, Phys. Rev. B 80, 104503 (2009).

73 Y. Zhang, B. Zhou, F. Chen, J. Wei, M. Xu, L. X. Yang, C. Fang, W. F. Tsai, G. H. Cao, Z. A. Xu, M. Arita, H. Hayashi, J. Jiang, H. Iwasawa, C.H. Hong, K. Shimada, H. Namatame, M. Taniguchi, J. P. Hu, and D. L. Feng,
arXiv:0904.4022 2 .

74 K. Ishida, Y. Nakai, and H. Hosono, J. Phys. Soc. Jpn. 78, 062001 (2009).

75 Q. Si, E. Abrahams, J. Dai, and J.-X. Zhu, New. J. Phys. 11, 045001 (2009).

76 M. Aichhorn, L. Pourovskii, V. Vildosola, M. Ferrero, O. Parcollet, T. Miyake, A. Georges, and S. Biermann, Phys. Rev. B 80, 085101 (2009).

77 M. J. Calderón, B. Valenzuela, and E. Bascones, New J. Phys. 11, 013051 (2009).

78 M. J. Calderón, B. Valenzuela, and E. Bascones, Phys. Rev. B 80, 094531 (2009).

79 P. M. R. Brydon, M. Daghofer, C. Timm, and J. van den Brink, Phys. Rev. B 83, 060501(R) (2011).

${ }^{80}$ E. Manousakis, J. Ren, S. Meng, and E. Kaxiras, Phys. Rev. B 78, 205112 (2008).

81 D. A. Papaconstantopoulos, M. J. Mehl, and M. D. Johannes, Phys. Rev. B 82, 054503 (2010).

82 A. M. Oleś, Phys. Rev. B 28, 327 (1983).

83 T. Nomura and K. Yamada, J. Phys. Soc. Jpn. 69, 1856 (2000).

${ }^{84}$ The tight-binding band structure of the two-orbital model is parameterized in terms of the nearest-neighbour hopping integral $t_{1}, \underline{43}$ which we here take as $t_{1}=1 \mathrm{eV}$. Although a smaller value of $t_{1}$ would give a more realistic bandwidth, this only results in a rescaling of the $T-U$ phase diagram and does not alter the underlying physics.

85 S. V. Borisenko, V. B. Zabolotnyy, D. V. Evtushinsky, T. K. Kim, I. V. Morozov, A. N. Yaresko, A. A. Kordyuk, G. Behr, A. Vasiliev, R. Follath, and B. Büchner, Phys. Rev. Lett. 105, 067002 (2010).

${ }^{86}$ S. H. Baek, H. J. Grafe, F. Hammerath, M. Fuchs, L. Harnagea, S. Wurmehl, J. van den Brink, and B. Büchner (unpublished).

87 Note that the this hole pocket is not visible in Ref. 48 as the "undoped" compound in this paper actually corresponds to a slight electron doping, $n=6.032$.

${ }^{88}$ M. Yi, D. H. Lu, J. G. Analytis, J.-H. Chu, S.-K. Mo, R.H. He, R. G. Moore, X. J. Zhou, G. F. Chen, J. L. Luo, N. L. Wang, Z. Hussain, D. J. Singh, I. R. Fisher, and Z.-X. Shen, Phys. Rev. B 80, 024515 (2009).

89 T. Yildirim, Phys. Rev. Lett. 102, 037003 (2009).

90 I.I. Mazin, M.D. Johannes, L. Boeri, K. Koepernik, and D.J. Singh, Phys. Rev. B 78, 085104 (2008).

91 V. Vildosola, L. Pourovskii, R. Arita, S. Biermann, and A. Georges, Phys. Rev. B 78, 064518 (2008).

92 C. Cao, P. J. Hirschfeld, and H.-P. Cheng, Phys. Rev. B 77, 220506(R) (2008).

${ }^{93}$ V. Barzykin and L. P. Gor'kov, Phys. Rev. B 79, 134510 (2009).

94 W. Lv, J. Wu, and P. Phillips, Phys. Rev. B 80, 224506 (2009).

95 C.-C. Chen, J. Maciejko, A. P. Sorini, B. Moritz, R. R. P. Singh, and T. P. Devereaux, Phys. Rev. B 82, 100504(R) (2010).

96 P. Hansmann, R. Arita, A. Toschi, S. Sakai, G. Sangiovanni, and K. Held, Phys. Rev. Lett. 104, 197002 (2010). 